ARTICLE

DOI: $10.1038 / s 41467-017-00761-8$

\title{
High-throughput RNA structure probing reveals critical folding events during early 605 ribosome assembly in yeast
}

Elena Burlacu1, Fredrik Lackmann², Lisbeth-Carolina Aguilar ${ }^{3}$, Sergey Belikov², Rob van Nues ${ }^{4}$, Christian Trahan ${ }^{3,5}$, Ralph D. Hector ${ }^{6}$, Nicholas Dominelli-Whiteley ${ }^{7}$, Scott L. Cockroft ${ }^{7}$, Lars Wieslander ${ }^{2}$, Marlene Oeffinger ${ }^{3,5,8} \&$ Sander Granneman (iD ${ }^{1}$

While the protein composition of various yeast $60 \mathrm{~S}$ ribosomal subunit assembly intermediates has been studied in detail, little is known about ribosomal RNA (rRNA) structural rearrangements that take place during early $60 \mathrm{~S}$ assembly steps. Using a highthroughput RNA structure probing method, we provide nucleotide resolution insights into rRNA structural rearrangements during nucleolar $60 \mathrm{~S}$ assembly. Our results suggest that many rRNA-folding steps, such as folding of 5.8S rRNA, occur at a very specific stage of assembly, and propose that downstream nuclear assembly events can only continue once 5.85 folding has been completed. Our maps of nucleotide flexibility enable making predictions about the establishment of protein-rRNA interactions, providing intriguing insights into the temporal order of protein-rRNA as well as long-range inter-domain rRNA interactions. These data argue that many distant domains in the rRNA can assemble simultaneously during early 605 assembly and underscore the enormous complexity of 605 synthesis.

\footnotetext{
${ }^{1}$ Centre for Synthetic and Systems Biology (SynthSys), University of Edinburgh, Edinburgh EH9 3BF, UK. 2 Department of Molecular Biosciences, The Wenner-Gren Institute, Stockholm University, SE-106 91 Stockholm, Sweden. ${ }^{3}$ Institut de Recherches Cliniques de Montréal RNP Biochemistry laboratory, Systems Biology Axis, Montréal, QC H2W 1R7, Canada. ${ }^{4}$ Institute of Cell Biology, University of Edinburgh, Edinburgh EH9 3FF, UK. ${ }^{5}$ Université de Montréal Département de Biochimie et Médecine Moléculaire Faculté de Médecine, Montréal," QC H3C 3J7, Canada. ${ }^{6}$ Institute of Neuroscience and Psychology, University of Glasgow, Glasgow G12 8QB, UK. ${ }^{7}$ EaStCHEM School of Chemistry, University of Edinburgh, Joseph Black Building, David Brewster Road, Edinburgh EH9 3FJ, UK. ${ }^{8}$ McGill University Division of Experimental Medicine Department of medicine, Montréal, QC H4A 3J1, Canada. Fredrik Lackmann and Lisbeth-Carolina Aguilar contributed equally to this work. Correspondence and requests for materials should be addressed to

S.G. (email: sgrannem@staffmail.ed.ac.uk)
} 
$\mathrm{R}$ ibosome assembly in the yeast Saccharomyces cerevisiae is a complex and energy-consuming process. It starts with the transcription of recombinant DNA by RNA polymerase I and the co-transcriptional packaging of nascent pre-ribosomal RNA (rRNA) into a $90 \mathrm{~S}$ complex ${ }^{1,2}$. Cleavage of the nascent transcript at site $\mathrm{A}_{2}$ (Fig. 1a) separates the large particle into precursors of the $40 \mathrm{~S}$ and $60 \mathrm{~S}$ subunits.

Assembly of the $60 \mathrm{~S}$ subunit involves processing of two rRNAs (5.8S and 25S rRNAs), and the incorporation of the third rRNA (5S rRNA) as a separate ribonucleoprotein complex ${ }^{3}$. Following cleavage of the nascent transcript at $\mathrm{A}_{2}$, the $27 \mathrm{SA}_{2}$ is converted into 27SB pre-rRNAs, either via cleavage at $A_{3}$ followed by exonucleolytic trimming to $\mathrm{B} 1_{\mathrm{S}}$ or by cleavage at $\mathrm{B} 1_{\mathrm{L}}$. The 27SB pre-rRNAs are cleaved at site $\mathrm{C}_{2}$ in the second internal transcribed spacer (ITS2) region, generating $7 \mathrm{~S}$ pre-rRNA and a $5^{\prime}$ extended $25 \mathrm{~S}$ precursor (25.5S) (Fig. 1a). These are subsequently processed in the nucleus, and undergo final maturation steps after their export to the cytoplasm to form mature 5.8S and 25S rRNAs, respectively ${ }^{4-6}$.

Pre-rRNA processing and folding requires the incorporation of many ribosomal proteins (r-proteins) and the activity of hundreds of assembly factors. Many of the r-proteins initially bind with low affinity, and their association becomes increasingly more stable as the assembly process proceeds ${ }^{7}$. This gradual assembly of r-proteins is regulated by ribosome assembly factors that chaperone their timely incorporation ${ }^{7}$, and make the assembly process more efficient by preventing misfolding or kinetic traps that could lead to the formation of defective ribosomes ${ }^{8}$.

Although the dynamics of the binding and dissociation of assembly factors has been studied in detail, still relatively little is known about the order of rRNA-folding steps, how assembly factors influence rRNA folding, and how this regulates the timely assembly of r-proteins. Recent biochemical and cryo electron microscopy (cryo-EM) studies have provided important insights into restructuring events in late pre-60S complexes ${ }^{9-14}$, however, still little is known about rRNA folding during the nucleolar stages. To address this, we performed high-throughput RNA structure probing analyses (ChemModSeq ${ }^{15}$ ) on purified nucleolar pre-60S particles isolated from the yeast $S$. cerevisiae. These data enabled us to generate a step-by-step model of the changes in rRNA flexibility during the nucleolar stages of $60 \mathrm{~S}$ assembly. Strikingly, our data indicate that the majority of the observed rRNA restructuring events take place during the conversion of $27 \mathrm{SA}_{2}$ to $27 \mathrm{SB}$. During this stage, the $5.8 \mathrm{~S}$ rRNA undergoes major structural rearrangements. These events coincide with the stable integration of r-proteins that are part of the polypeptide exit tunnel and the release of assembly factor Rrp5 that plays an important role in chaperoning rRNA folding ${ }^{16}$. Furthermore, we provide the first comprehensive structural analysis of ITS2 secondary structure, which demonstrate that ITS2 forms a highly compact structure, consistent with recent phylogenetic analyses ${ }^{17}$. Finally, comparing our nucleotide flexibility maps with the crystal structure of the $60 \mathrm{~S}$ subunit enabled us to make predictions about the timing of the formation of $r$ protein-rRNA interactions as well as the formation of many longrange inter-domain interactions within the 60S subunit rRNAs.

Collectively, the results provide insights into the temporal order of rRNA restructuring events during $60 \mathrm{~S}$ assembly and present a very useful resource for the design of more focussed functional analyses.

\section{Results}

Purification of pre-60S intermediates. To measure RNA structural differences between large subunit (LSU) pre-rRNA species, we purified pre-60S particles using TAP-tagged bait proteins previously shown to be incorporated in complexes containing 35S (Mrd1), 27SA 2 (Rrp5), and 27SB (Nsa2) rRNAs (Fig. 1a, b $)^{18-20}$. After IgG affinity purification, co-precipitated pre-rRNAs were resolved on an agarose gel and the RNAs of interest were excised from the gel and purified. To assess the homogeneity of the purified pre-rRNA species, primer extension analysis was performed (Fig. 1b). The data show that Rrp5-TAP predominantly co-precipitated $27 \mathrm{SA}_{2}$ pre-rRNA (Fig. 1b, lane 1) and Nsa2-TAP almost exclusively co-precipitated $27 \mathrm{SB}$ pre-rRNA (Fig. 1b, lane 2). As TAP purifications with 90S-associated factors did not generate sufficient $35 \mathrm{~S}$ pre-rRNA for high-throughput pre-rRNA structure probing analyses (data not shown), we used a TAP-tagged Mrd1 mutant strain $(\operatorname{Mrd} 1 \Delta 5)$ that is kinetically delayed in $35 \mathrm{~S}$ pre-rRNA processing but does not noticeably affect $27 \mathrm{~S}$ pre-rRNA levels (Supplementary Fig. 1a, b) 20,21 .

We also performed label-free quantitative mass spectrometry (MS) on the 605 pre-ribosomal particles purified using Rrp5 and $\mathrm{Nsa} 2$ as baits as well as $80 \mathrm{~S}$ ribosomes, isolated using the translation initiation factor Fun12 (Fig. 1c-e, Supplementary Fig. 1c-e). We did not analyze the proteome of the $\operatorname{Mrd} 1 \Delta 5$ particles as LSU assembly factors are likely highly under-represented in this complex, making the direct comparison with $60 \mathrm{~S}$ particles complicated. The pre-60S MS data (Supplementary Data 1) are in excellent agreement with previous proteomic analyses of Rrp5- and Nsa2-associated complexes $14,22-24$. Early 90S-associated assembly factors only co-precipitated with Rrp5, consistent with Rrp5 being part of both the $90 \mathrm{~S}$ pre-ribosomes and early pre-60S intermediates ${ }^{25}$, while Nsa2 associates with $27 \mathrm{SB}$ pre-rRNA containing pre-60S intermediates $^{26}$.

Conversion of $27 \mathrm{SA}_{3}$ to $27 \mathrm{SB}$ requires 12 proteins that are interdependent for their assembly, and which are referred to as $\mathrm{A}_{3}$ factors (Fig. 1a) ${ }^{27}$. Cleavage of $27 \mathrm{SB}$ at site $\mathrm{C}_{2}$ within ITS2 requires a set of assembly factors termed B-factors ${ }^{28}$ (Fig. 1a). Interestingly, most $\mathrm{A}_{3}$ factors (Nop7, Erb1, Ytm1, Rlp7, Cic1, and Nop15) were readily detectable in both Rrp5 and Nsa2 particles (Fig. 1c, d), suggesting that these proteins do not dissociate once $27 \mathrm{SB}$ has been generated. Notably, the $\mathrm{A}_{3}$ factors were significantly enriched over the $B$ factors in the Rrp5 pre-ribosomes (Fig. 1c), while in the Nsa2 particle, $\mathrm{A}_{3}$ and $\mathrm{B}$ factors were present in comparable amounts (Fig. 1d). Assembly of the $\mathrm{B}$ factors takes place in two stages ${ }^{28}$; some $\mathrm{B}$ factors bind to the early $35 \mathrm{~S}$ and $27 \mathrm{SA}_{2}$ pre-rRNA containing pre-ribosomes (Nop2, Nip7, Rpf2, Rrs1, Rlp24, Nog1, Tif6, Mak11, Spb4, and Dpb10), while others bind just prior to $\mathrm{C}_{2}$ cleavage (Nsa2 and Nog2). Consistent with this, the early B factors were detected in both the Rrp5 and Nsa2 pre-60S intermediates, whereas the later B factors were predominantly present in the Nsa2 particles (Fig. 1c, d).

Further comparison between the r-protein content of the Fun12-, Rrp5-, and Nsa2-associated particles indicated that the purified pre-60S intermediates contained a similar r-protein composition, although not all r-proteins could be detected with high confidence due to a dynamic range detection limit of the instruments. Note that in this manuscript, we combine both the old and new nomenclature for the yeast $r$-proteins ${ }^{29,30}$. The $r$ proteins that are readily detected in very early $60 \mathrm{~S}$ pre-ribosomes, such as L3/uL3 and L4/uL4 were identified in equivalent amounts in Rrp5 and Nsa2 pre-60S intermediates. Some r-proteins were slightly more abundant in the Nsa2 particle compared to the Rrp5 particle. This modest imbalance could be explained by the fact that r-proteins become more stably incorporated into pre-ribosomes as the maturation progresses $^{7,31}$. 
a

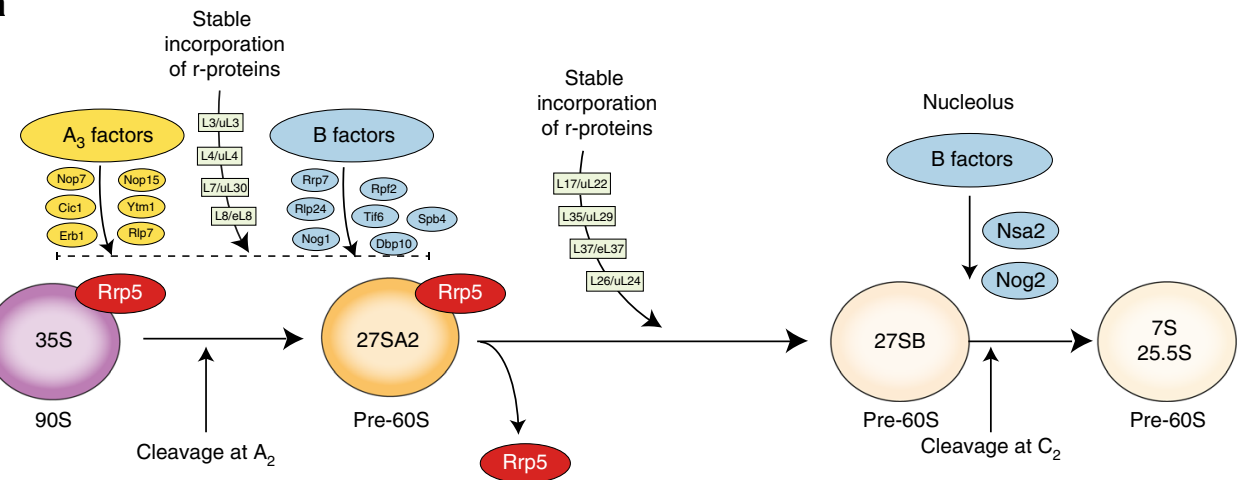

b

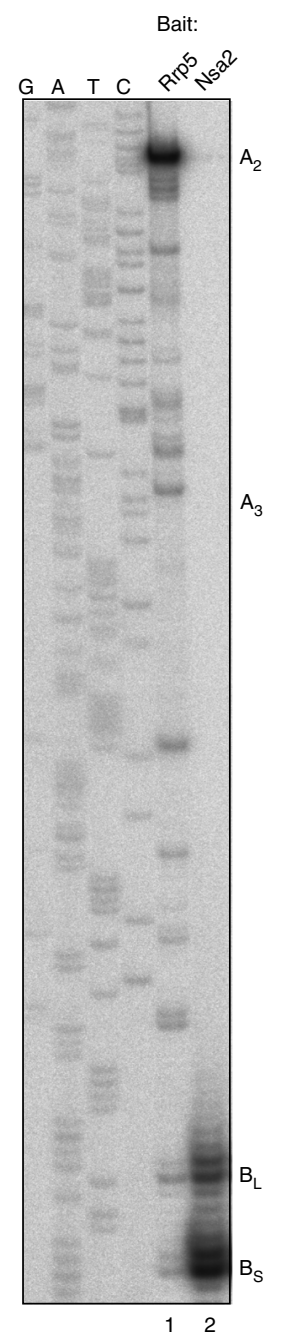

C

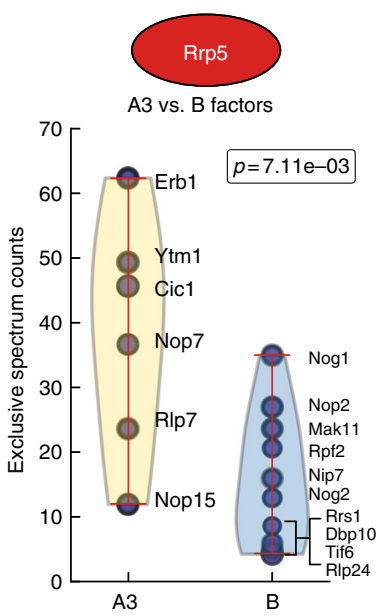

d

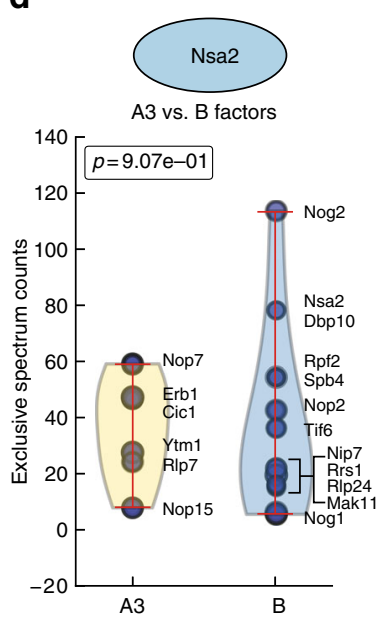

e

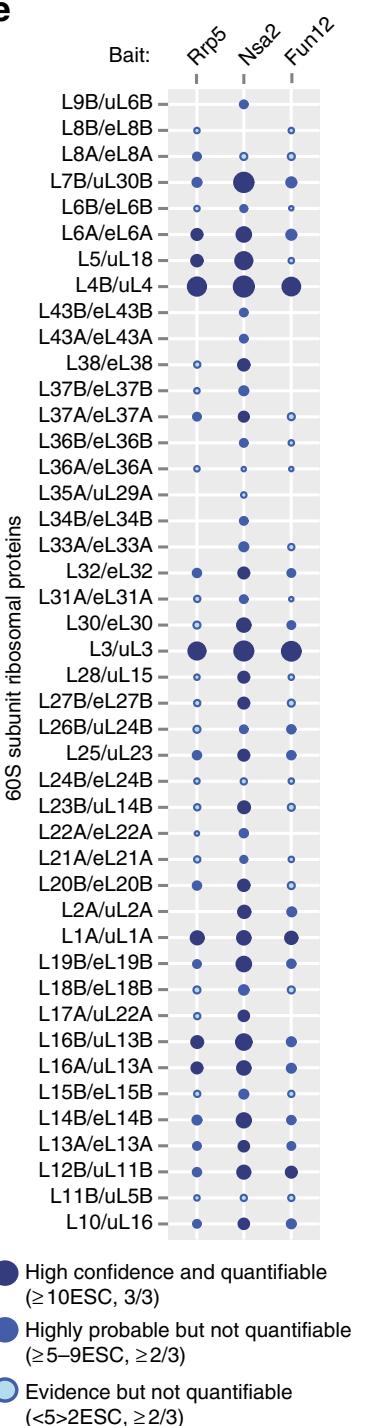


In conclusion, the purified pre-60S complexes showed the expected protein composition, providing a good basis for our structure probing analyses.

ChemModSeq analyses of purified pre-60S ribosomes. To obtain pre-rRNA secondary structure information, the affinity purified pre-ribosomes were treated in vitro with the SHAPE chemical 1-methyl-7-nitroisatoic anhydride (1M7). Subsequently, the pre-rRNAs were resolved on agarose gels to remove contaminants and gel purified ${ }^{15}$. 1M7 generically reacts with all four nucleotides in flexible (mostly single-stranded) regions and acylates the $2^{\prime} \mathrm{OH}$ of the ribose ${ }^{32}$. To be able to assess the effect of protein binding events on pre-rRNA flexibility, we chemically probed pre-rRNAs from proteinase $\mathrm{K}(\mathrm{PK})$-treated particles (Fig. 2a, Supplementary Fig. 1f). The rRNA regions bound by proteins can become more reactive to $1 \mathrm{M} 7$ in the deproteinized particles, in particular, those regions where proteins contact areas of single-stranded rRNA. This provided us with a unique approach to monitor the proposed gradual stabilization of $r$ protein association with pre-ribosomes 7,31

The purity and integrity of the $27 \mathrm{~S}$ pre-rRNA samples were analyzed using a bioanalyzer (27S pre-rRNAs; Fig. 2a) or agarose gels (35S pre-rRNA; Supplementary Fig. 1b, lane 7). Primer extension was performed to assess whether the samples were sufficiently modified (data not shown). Subsequently, ChemModSeq libraries were prepared from these samples and high-throughput sequenced. ChemModSeq data from two biological replicates were pooled to increase the total coverage, followed by calculation of normalized 1M7 reactivities ${ }^{33}$. The $1 \mathrm{M} 7$ reactivity data is provided in Supplementary Data 2. Pearson correlation analysis revealed a positive correlation between $35 \mathrm{~S}$, $27 \mathrm{SA}_{2}$, and $27 \mathrm{SB}$ data (Fig. $2 \mathrm{~b}-\mathrm{d}$ ). However, the $35 \mathrm{~S}$ and $27 \mathrm{SA}_{2}$ data were considerably more highly correlated than either $35 \mathrm{~S}$ or $27 \mathrm{SA}_{2}$ and $27 \mathrm{SB}$. These results suggest that the $27 \mathrm{~S}$ pre-rRNA undergoes major changes in RNA flexibility during the conversion of the $27 \mathrm{SA}_{2}$ to $27 \mathrm{SB}$ particle and that the pre-rRNA structure in the $35 \mathrm{~S}$ and $27 \mathrm{SA}_{2}$ particles is quite similar. The highest correlation between any two data sets in our analysis was found for deproteinized $27 \mathrm{SA}_{2}$ and $27 \mathrm{SB}$ pre-rRNAs (Fig. 2e), indicating that many of the observed changes in reactivity between $27 \mathrm{SA}_{2}$ and $27 \mathrm{SB}$ may be due to RNA-protein interactions.

To determine how the structural information provided by the $1 \mathrm{M} 7$ probing experiments compares to the crystal structure of the $25 \mathrm{~S}$ rRNA in the $60 \mathrm{~S}$ subunit $^{34}$, for each domain in the $25 \mathrm{~S}$ rRNA, we calculated area under ROC (receiver operating characteristic) curves using a binary classifier that only included nucleotides that were single stranded and not contacted by $r$-proteins in the crystal structure. As expected, these data indicate that the very early pre-60S intermediates analyzed in this study are structurally distinct from the mature 60S subunit (Supplementary Fig. 2). These data further indicate that domains I, II, and VI in the $35 \mathrm{~S}$ and $27 \mathrm{SA}_{2}$ particles, and domains II, III, and $\mathrm{V}$ in the $27 \mathrm{SB}$ particle, are more similar to the crystal structure than other domains in these pre-60S particles.

Gradual assembly of r-proteins into $60 \mathrm{~S}$ pre-ribosomes. To identify statistically significant differences in pre-rRNA nucleotide flexibility between the different particles, we employed the previously described $\triangle$ SHAPE algorithm ${ }^{35}$. SHAPE reactivities from different particles were subtracted and reactivities were averaged over a three-nucleotide sliding window to reduce local signal fluctuation. To be considered statistically significant, the difference in SHAPE reactivity ( $\triangle$ SHAPE) had to be at least 1 standard deviation higher than the mean $\triangle$ SHAPE values $^{35}$. Because the $35 \mathrm{~S}$ and $27 \mathrm{SA}_{2}$ data showed very similar SHAPE reactivity patterns, we focussed our analyses on the differences observed between $35 \mathrm{~S}, 27 \mathrm{SA}_{2}$, and the $27 \mathrm{SB}$ data (Fig. $3 \mathrm{a}, \mathrm{b}$ ). The $\triangle \mathrm{SHAPE}$ analyses confirmed that the $27 \mathrm{SB}$ particle showed a markedly different SHAPE reactivity profile compared to the other samples analyzed (Fig. 3b). Almost all of the 25S rRNA domains appeared to undergo conformational changes during the nucleolar stages of $60 \mathrm{~S}$ assembly (Fig. 3a, b). The most striking observations were the increase in nucleotide flexibility in the $3^{\prime}$ end region of the 27SB pre-rRNA (domains IV-VI) and the fact that the $5.8 \mathrm{~S}$ showed significantly higher SHAPE reactivity in the $35 \mathrm{~S}$ and $27 \mathrm{SA}_{2}$ particles. The latter suggests that the $5.8 \mathrm{~S}$ region undergoes major conformational changes during the conversion of $27 \mathrm{SA}_{2}$ to $27 \mathrm{SB}$ (also see below). In contrast, relatively few changes in flexibility were observed in ITS2 (Fig. 3b).

To be able to assess which changes in flexibility could be explained by protein-binding events, we next compared the pre-ribosome SHAPE data to the data obtained from the PK-treated samples (Fig. 3c). When we discuss PK-sensitive nucleotides, we refer to positions that showed significant $\triangle$ SHAPE values when comparing data from $35 \mathrm{~S}$ or $27 \mathrm{SA}_{2}$ to 27SB particles, however, this change in flexibility was reversed in the PK-treated 27SB data. When we discuss PK-insensitive sites, we refer to positions that showed significant $\triangle$ SHAPE values between $35 \mathrm{~S}-27 \mathrm{~A}_{2}$ and $27 \mathrm{SB}$ particles, which were not reversed by $\mathrm{PK}$ treatment of the $27 \mathrm{SB}$ particles. The majority of the nucleotides that showed a decrease in SHAPE reactivity in 27SB particles were PK-insensitive. These data suggest that these changes were unlikely the result of protein-binding events. The majority of these sites was located in domains 0-III (Fig. 3c), indicating that these domains fold into a more compact structure in the nucleolus. In contrast, most of the sites that showed increased flexibility in the 27SB particles were PK-sensitive and concentrated in domains III-V. These data suggest that RNA-binding proteins that interact with the 27SB particle (such as assembly factors or r-proteins) increase the flexibility of these domains. Interestingly, a fraction of these sites also overlapped with r-protein-binding sites (Fig. 3c; r-proteinbinding sites, increase in flexibility).

For a number of nucleotides, we observed a decrease in flexibility in 27SB particles, and these changes were PK-sensitive (Fig. 3c; decrease in flexibility and PK-sensitive). We speculate that gradual assembly of r-proteins that bind with low affinity to early $60 \mathrm{~S}$ pre-ribosomes, but become more stably bound in later intermediates, could explain some of these changes $3,7,24,36,37$, although alternative explanations are possible (see Discussion). We refer to these sites as PKSRP (PK-sensitive SHAPE reactive sites bound by r-proteins). Interestingly, most of the PKSRP sites were mainly located/concentrated on the solvent-exposed interface (in particular in domain I) of the $60 \mathrm{~S}$ subunit, but were under-represented on the subunit interface (which includes domains III and IV), the central protuberance, and the feet (Fig. 3d, Supplementary Data 3, Supplementary Movie 1).

Formation of long-range inter-domain interactions. Several of the PKSRP sites are contacted by the same ribosomal protein in the mature 60S subunit, but lie in different structural domains. Some of these nucleotides showed a decrease in reactivity during the conversion of $35 \mathrm{~S}$ to $27 \mathrm{SA}_{2}$ and $27 \mathrm{SA}_{2}$ to $27 \mathrm{SB}$. We interpret these changes as gradual stabilization of $r$-protein binding and formation or stabilization of long-range and/or inter-domain interactions. Some examples are discussed below and a complete overview can be found in Supplementary Data 3. The locations of the PKSRP sites suggest that interactions between domains 


\section{a}
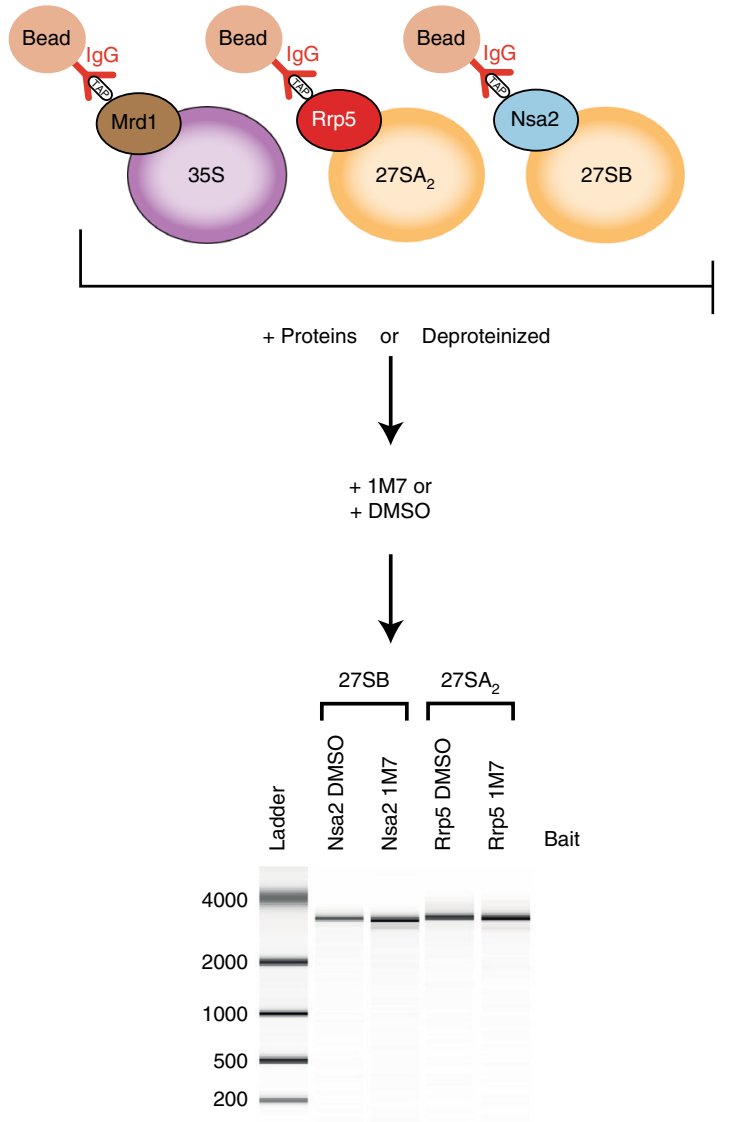

L $\begin{array}{llll}1 & 2 & 3 & 4\end{array}$

Pre-60s

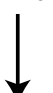

ChemModSeq library preparation
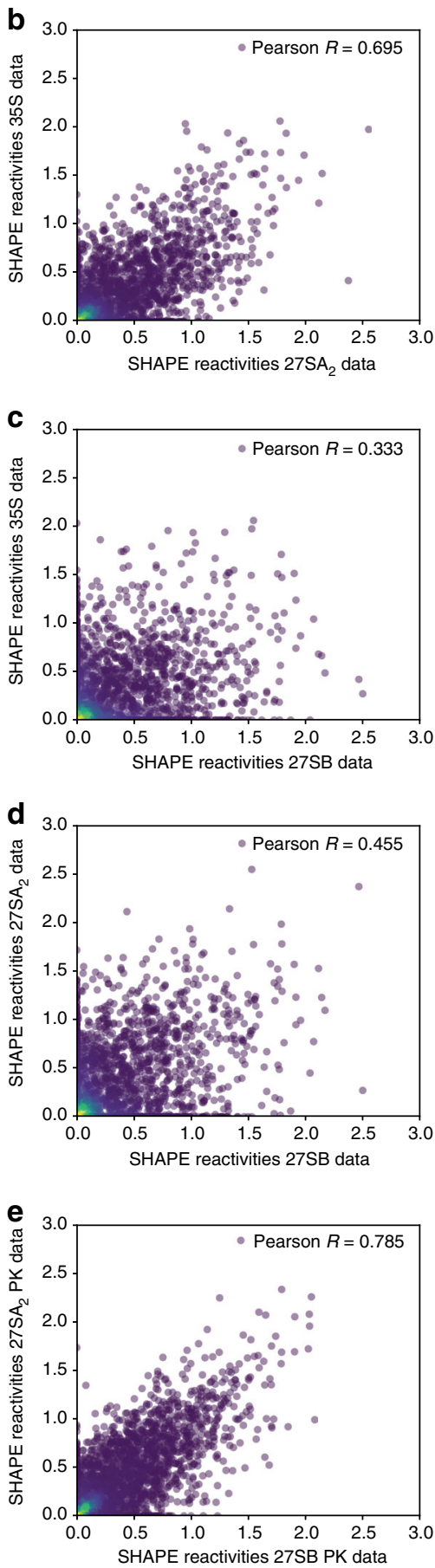

Fig. 2 ChemModSeq experimental workflow and data analysis. a Particles containing the 35S, 27SA 2 , and 27SB pre-rRNAs were purified using TAP-tagged Mrd1, Rrp5, or Nsa2 as baits, respectively. Chemical probing was performed on the purified particles as well as PK-treated particles. As controls for natural primer extension stops, we also incubated purified particles and PK-treated particles with the $1 \mathrm{M7}$ solvent (DMSO). The rRNAs were gel purified and their integrity was assessed on a bioanalyzer chip. b-e Many conformational changes take place between the $27 \mathrm{~A}_{2}$ and $27 \mathrm{SB}$ particles. Shown are scatter plots that compare normalized SHAPE reactivity values of the $35 \mathrm{~S}$ data to $27 \mathrm{SA} \mathrm{A}_{2}$ and $27 \mathrm{SB}$ data (b) and (c) and $27 \mathrm{SA} \mathrm{A}_{2}$ compared to $27 \mathrm{SB}$ data (d). Data generated from deproteinized particles is shown in $\mathbf{e}$. The similarity between the data sets was determined by calculating Pearson correlation coefficients

I, II, V, and VI are formed by L4/uL4, L17/uL22, and L42/eL43 during the conversion of $35 \mathrm{~S}$ to $27 \mathrm{SB}$ pre-rRNA (Fig. 4a). Similarly, we predict that several long-range interactions between domains III and V are formed by L2/uL2, L34/uL34, and L43/eL43 in the nucleolus (Fig. 4b). Interestingly, in the latter cases, the $\mathrm{r}$-proteins initially bind domain $\mathrm{V}$ during the conversion of $35 \mathrm{~S}$ to $27 \mathrm{SA}_{2}$ and then bind domain III in the 27SB particle.
Collectively, the data shown here indicate that rRNA-folding steps within many domains of the $25 \mathrm{~S}$ rRNA region take place in the nucleolus, suggesting that many distant domains in the pre-rRNA could fold around the same time.

Restructuring of $5.8 \mathrm{~S}$ during the conversion of $27 \mathrm{SA}_{2}$ to $27 \mathrm{SB}$. The $\triangle$ SHAPE analyses as well as follow-up primer extension 

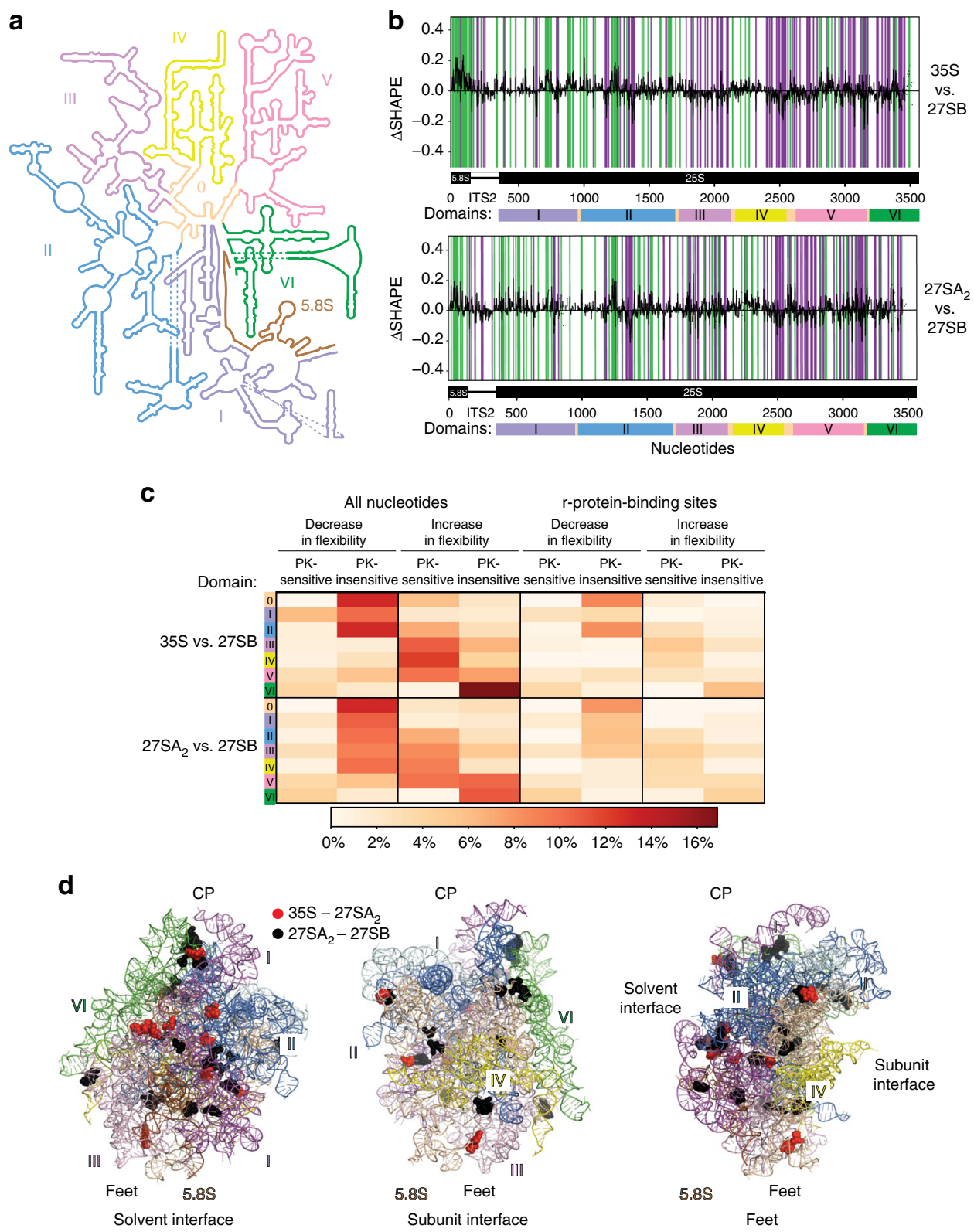

Fig. 3 Folding of $25 \mathrm{~S}$ rRNA domains. a Secondary structure model for the $25 \mathrm{~S}$ and $5.8 \mathrm{~S}$ rRNAs generated using RiboVision ${ }^{64}$. The individual domains are colored. $\mathbf{b}$ Differential SHAPE ( $\triangle \mathrm{SHAPE}$ ) analysis of $35 \mathrm{~S}, 27 \mathrm{SA}$, and $27 \mathrm{SB}$ particles. The histograms show a comparison between $35 \mathrm{~S}$ and $27 \mathrm{SB}$ data and $27 \mathrm{SA}_{2}$ compared to $27 \mathrm{SB}$ data. If a region is highlighted in green, then it is more reactive in the $35 \mathrm{~S}$ (top) or $27 \mathrm{SA} \mathrm{A}_{2}$ (bottom) data. If a region is highlighted in purple, it is more reactive in the 27SB data. $\triangle$ SHAPE reactivities were calculated as using code developed by the Weeks lab ${ }^{36}$. c The heat map indicates the percentage of nucleotides in each 25S rRNA domain (indicated on the left side of each domain) that showed a decreased or an increase reactivity when comparing the various particles ( $35 \mathrm{~S}$ vs. $27 \mathrm{SB}$ and $27 \mathrm{SA} \mathrm{A}_{2}$ vs. $27 \mathrm{SB}$ ) as well as whether these changes were sensitive or insensitive to PK treatment. Shown are the results for all the nucleotides as well as the nucleotides that overlapped with r-protein-binding sites in the $60 \mathrm{~S}$ crystal structure ${ }^{35}$. $\mathbf{d}$ Overview of the PKSRP sites in the three-dimensional structure of the $25 \mathrm{~S} r R N A^{35}$. Most of the proposed protein-rRNA interactions are concentrated on the solvent interface, with fewer PKSRP sites identified on the subunit interface, central protuberance (CP), and feet. Protein-binding sites were defined according to the crystal structure of the ribosome $\mathrm{e}^{35}$

analyses indicated that the $5.8 \mathrm{~S}$ region undergoes considerable structural rearrangements during the conversion of $35 \mathrm{~S}$ to $27 \mathrm{SB}$ pre-rRNA (Fig. 3b, Supplementary Fig. 3). Closer inspection of modification patterns generated by both methods revealed that the major changes in RNA flexibility in this region occur specifically during the conversion of $27 \mathrm{SA}_{2}$ to $27 \mathrm{SB}$ (Fig. 5a, compare lanes 2 and 3, Supplementary Fig. 3a-c). The observed restructuring likely coincides with binding of r-proteins to this region, which is indicated by the fact that some of their nucleotide-binding sites in the $5.8 \mathrm{~S}$ became less flexible in the $27 \mathrm{SB}$ pre-rRNA than in the earlier precursors (Fig. 5a; Supplementary Fig. 3), and that this reduction in flexibility was (at least in part) reversed in the deproteinized samples. Thus, our data suggest that the folding of some $5.8 \mathrm{~S}$ regions may coincide with the binding of r-proteins L26/uL24, L35/uL29, and L37/eL37 to the 5.8S in the 27SB pre-rRNA.

Nop12 has previously been reported to be important for the formation of $\mathrm{H}^{38}$. The Nop12-binding sites in $5.8 \mathrm{~S}$ became less 
a
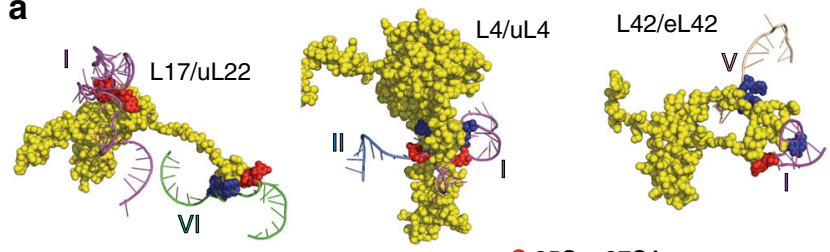

b
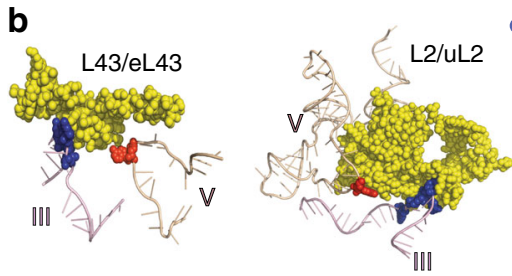

- $35 \mathrm{~S}-27 \mathrm{SA}_{2}$

$27 \mathrm{SA}_{2}-27 \mathrm{SB}$

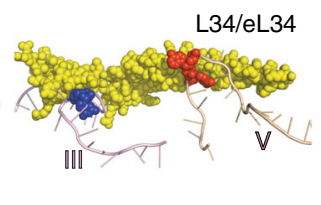

Fig. 4 Models of inter-domain interactions formed during nucleolar stages of 605 assembly. The yellow spheres indicate $r$-proteins. Predicted protein-rRNA interactions formed during the conversion of $35 \mathrm{~S}$ of $27 \mathrm{SA}_{2}$ and the conversion of $27 \mathrm{SA}_{2}$ to $27 \mathrm{SB}$ are indicated with red and blue dots, respectively. Protein-binding sites were defined according to the crystal structure of the ribosome ${ }^{35}$. a Binding of L17/uL22 L4/uL4 and L42/eL42 to domains I (purple), V (wheat), and VI (green) of the $25 \mathrm{~S}$ during the conversion of $35 \mathrm{~S}$ to $27 \mathrm{SB}$ pre-rRNA. b Sequential binding of L43/eL43, L2/uL2, and L34/eL34 brings domains III (pink) and V (wheat) in close proximity

flexible in the 27SB particles (Fig. 5a), but this flexibility was restored upon deproteinization. These data therefore suggest that Nop12-dependent formation of $\mathrm{H} 5$ may take place during the conversion of $27 \mathrm{SA}_{2}$ to $27 \mathrm{SB}$.

Helices $\mathrm{H} 2, \mathrm{H} 3$, parts of $\mathrm{H} 4$ and $\mathrm{H} 10$, which are formed by base-paring interactions between $5.8 \mathrm{~S}$ and the $25 \mathrm{~S}$ (Fig. 5b), appeared to be mostly structured in all three precursors (Fig. 5a; Supplementary Data 2) and did not show any statistically significant differences in reactivity between the $35 \mathrm{~S}$ and $27 \mathrm{SB}$ data (Fig. 5c, black nucleotides). These results indicate that many 5.8S-25S base-pairing interactions form early, possibly co-transcriptionally. Consistent with this idea, the $25 \mathrm{~S}$ nucleotides that base-pair with these $5.8 \mathrm{~S}$ regions generally reacted poorly with $1 \mathrm{M} 7$ and did not show significant changes in flexibility in all the particles analyzed (Fig. 5c, Supplementary Data 2). Thus, formation of $5.8 \mathrm{~S}-25 \mathrm{~S}$ base-pairing interactions precedes folding of $5.8 \mathrm{~S}$.

Other helices in $5.8 \mathrm{~S}$ appeared to be unstructured in the $35 \mathrm{~S}$ and $27 \mathrm{SA}_{2}$ particles but became significantly less flexible in the 27SB particle. Nucleotides in $\mathrm{H} 5-\mathrm{H} 8$ were significantly more reactive to $1 \mathrm{M} 7$ in $35 \mathrm{~S}$ and $27 \mathrm{SA}_{2}$ particles compared to $27 \mathrm{SB}$ (Fig. 5c; Supplementary Data 2).

As we were unable to probe later pre-60S intermediates (see Discussion), we could not determine whether additional 5.8S restructuring occurs in later assembly intermediates. However, a recent cryo-EM study on a nuclear Nog2-associated pre-60S particle, which has a similar protein composition as the Nsa2 particle analyzed here, revealed that the structure of the $5.8 \mathrm{~S}$ region is almost identical to that of $5.8 \mathrm{~S}$ rRNA in the mature ribosome ${ }^{12,34}$ (Supplementary Fig. 4). Therefore, it is reasonable to conclude that in the Nsa2 particle, the $5.8 \mathrm{~S}$ rRNA is almost completely folded and that therefore the majority of restructuring events takes place during the conversion of $27 \mathrm{SA}_{2}$ to $27 \mathrm{SB}$ and involves formation of additional $r$-protein-rRNA interactions.

ITS2 and flanking regions form compact structures. Our structure probing data show that in contrast to the $5.8 \mathrm{~S}$ region,
ITS2 is generally highly structured and does not appear to undergo any major changes in secondary structure during conversion of $35 \mathrm{~S}$ to $27 \mathrm{SB}$ (Figs. $3 \mathrm{~b}$ and $6 \mathrm{~b}, \mathrm{c}$ ). We compared our data to three previously proposed ITS2 secondary structure models (Fig. 6a): the "ring" model centered on phylogenetic analysis ${ }^{39}$, the "hairpin" model, largely based on enzymatic and chemical RNA structure probing ${ }^{40}$, and a structure that we refer to as the "ring-pin model"17. The latter is mostly based on phylogenetic data and is a hybrid of the ring and hairpin models (Fig. 6a).

In general, our ChemModSeq data (Fig. 6b) and follow-up primer-extension data (Fig. 6c, Supplementary Fig. 5) agree best with the ring-pin structure, which we also found to be consistent with previously published mutagenesis data ${ }^{41-43}$ (Supplementary Fig. 6).

The structure probing data support the proposed stem III in the ring-pin model, involving many A-U base-pairs (Fig. 6a-c). However, we note that although many nucleotides in region II are drawn as single-stranded nucleotides (Fig. 6a), it appears that they are less flexible compared to other loop regions within ITS2. A recent pre-60S cryo-EM structure uncovered the structure of three $\mathrm{A}_{3}$ factors (Cic1, Rpl7, and Nop15) bound to fragments of ITS $2^{12}$, essentially covering almost completely regions I and II. This may explain why the probing data showed low flexibility in this region (Fig. 6d). We did observe a strong reduction in reactivity in the flexibility of $\mathrm{U}_{6}$ near the $5^{\prime}$ end of ITS2 in the deproteinized data, reminiscent of what was observed in cells depleted of $\mathrm{A}_{3}$ factors ${ }^{44,45}$ (also see Discussion). In the cryo-EM structure, this nucleotide appears to be contacted by Cicl and $\mathrm{Rlp} 7^{12}$, apparently stabilizing a conformation that favors $1 \mathrm{M} 7$ modification. The observed reduction in $1 \mathrm{M} 7$ reactivity in deproteinized samples suggests that the high flexibility of this nucleotide is indeed associated with $\mathrm{Cicl}$ and Rlp7 binding to the $5^{\prime}$ end of ITS2.

The 27SB particle reproducibly showed higher reactivities in ITS2 in the ChemModSeq data, particularly in the 100-200 region (Fig. 6b). Although this may indicate that ITS2 undergoes structural rearrangements in the Nsa2 particle, these sites were all located in bulge or loop regions (Fig. 6a), suggesting that these single-stranded regions are more effectively probed in the Nsa2 particle. This may be related to the differences in protein composition between the Nsa2 and Rrp5 particles.

\section{Discussion}

Ribosome synthesis is a complex and dynamic process that involves the regulated folding of rRNA and hierarchical assembly of r-proteins. To obtain a step-by-step picture of the changes in rRNA folding during $60 \mathrm{~S}$ maturation, we isolated the $35 \mathrm{~S}, 27 \mathrm{SA}_{2}$, and $27 \mathrm{SB}$ pre-rRNA intermediates from native pre-60S particles and measured nucleotide flexibilities using a high-throughput RNA structure probing method (ChemModSeq ${ }^{15}$ ). This allowed us to generate a global map of changes in rRNA flexibility during the early nucleolar stages of pre-60S maturation, providing novel insights into rRNA restructuring events as well as the establishment and timing of long-range interactions that take place during nucleolar stages of assembly. The work described here provides a solid basis for the future design of more focussed functional and structural studies.

We show that using specific TAP-tagged baits and gel purification, we can effectively separate the $35 \mathrm{~S}, 27 \mathrm{SA}_{2}$, and $27 \mathrm{SB}$ pre-rRNAs. This was supported by primer extension, bioanalyzer, and agarose gel analyses (Figs. $1 \mathrm{~b}$ and $2 \mathrm{a}$ and Supplementary Fig. 1b), thus arguing for the presence of a single RNA species in each of the structural probing analyses. However, specific affinity purified pre-rRNA particles that contain a distinct 
a

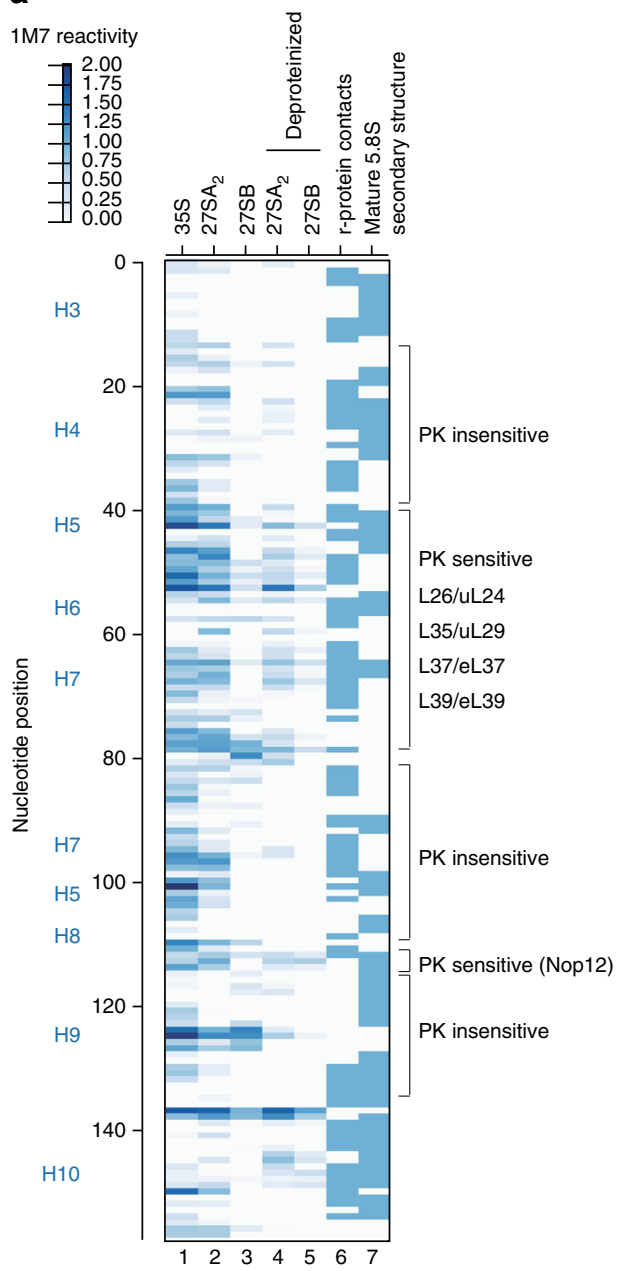

b

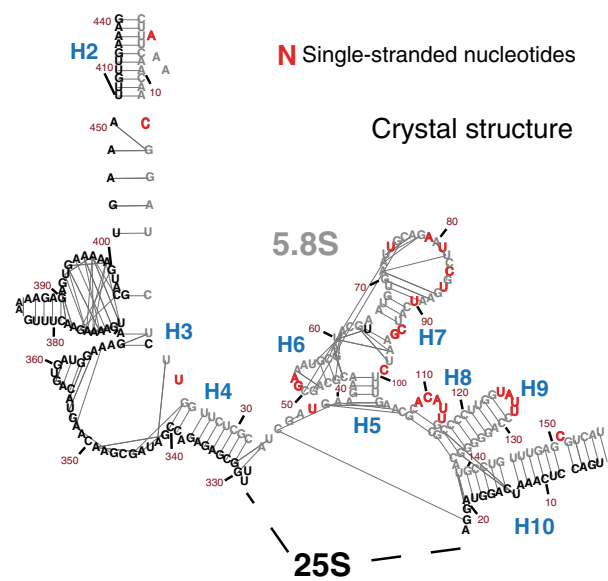

C

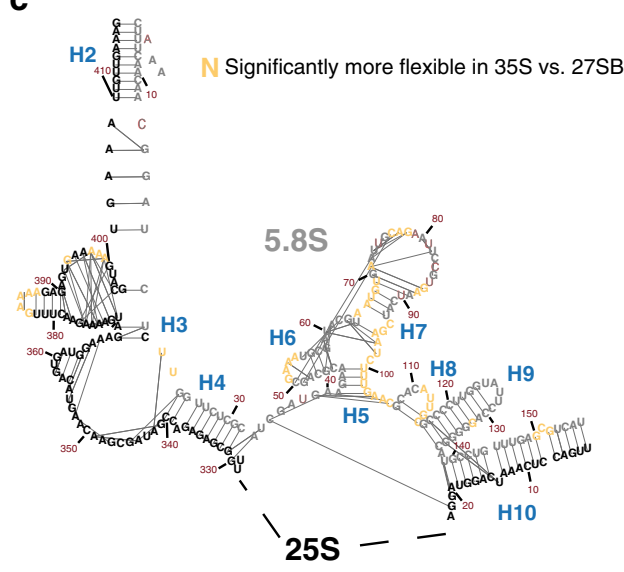

Fig. 5 The $5.8 \mathrm{~S}$ region undergoes a major restructuring event during the conversion of $27 \mathrm{SA} \mathrm{A}_{2}$ to $27 \mathrm{SB}$ pre-rRNA. a Heat map representing the ChemModSeq $1 \mathrm{M} 7$ reactivities. Each block indicates a single nucleotide, the darker the block the higher the $1 \mathrm{M} 7$ reactivity. The blue blocks in lane 6 indicate r-protein-binding sites ${ }^{35}$. Lane 7 represents the nucleotides that are base-paired (blue blocks) or single-stranded (white blocks) in the mature 5.8S rRNA crystal structure ${ }^{35}$. b Secondary structure of the $5.8 \mathrm{~S}$ rRNA. Black lines indicate base-pairing interactions observed in the yeast $80 \mathrm{~S}$ crystal structure. The red nucleotides are single-stranded in the crystal structure. c Overview of $\triangle \mathrm{SHAPE}$ analysis in the $5.8 \mathrm{~S}$ region. Yellow-colored nucleotides showed significantly higher SHAPE values in $35 \mathrm{~S}$ compared to the 27SB particle data

pre-rRNA species could naturally differ in protein content and structure as these particles go through various assembly states. Thus, our structure probing data likely show averaged 1M7 reactivity profiles of these pre-rRNA particles. Nevertheless, our current data as well as our previously published work ${ }^{15}$ clearly demonstrate that conformational changes can effectively be detected in purified pre-ribosomes using chemical probing. Most of the restructuring we detected here are likely rate-limiting events that are triggered by the release of ribosome assembly factors, binding of r-proteins, or the activity of energy-dependent enzymes.

We also wished to compare the pre-rRNA structures of nucleolar particles with later (nuclear) assembly intermediates. However, because the pre-rRNAs in these particles $\left(5^{\prime}\right.$-extended $25 \mathrm{~S}$ species) could not be separated from contaminating $25 \mathrm{~S}$ rRNA by gel electrophoresis, we were unable to perform these experiments. Generating sufficient quantities of highly pure $35 \mathrm{~S}$ pre-rRNA for high-throughput RNA structure probing was very challenging. In order to obtain sufficient $35 \mathrm{~S}$ pre-rRNA, we utilized a TAP-tagged Mrd1 mutant strain in which one of the RNA-binding domains was deleted (Mrd1 $\Delta 5$-TAP). Although
pre-40S synthesis is impaired in the Mrd1 $\Delta 5$ mutant, leading to the accumulation of $35 \mathrm{~S}$ pre-rRNA, it remains associated with 90S pre-ribosomes and the 25S rRNA levels are similar to those of wild-type cells indicating that LSU processing is unaffected ${ }^{20,} 21$ (Supplementary Fig. 1a, b). We therefore argue that the $35 \mathrm{~S}$ pre-rRNA purified from the Mrd $1 \Delta 5$ mutant can be efficiently processed into mature $25 \mathrm{~S}$ and $5.8 \mathrm{~S}$ rRNA. As $35 \mathrm{~S}$ pre-rRNA accumulates in this strain, we cannot exclude that its folding kinetics differ from $35 \mathrm{~S}$ pre-rRNA purified from a wild-type strain. However, we were able to purify several nanograms of 1M7-probed 35S pre-rRNA from the Rrp5-TAP strain sufficient for primer extension analysis (Supplementary Fig. 7). We did not observe any noticeable differences in reactivity pattern in the $5.8 \mathrm{~S}$ region (the region where many changes in RNA flexibility were observed) between the $35 \mathrm{~S}$ pre-rRNA purified from Rrp5-TAP and $\operatorname{Mrd} 1 \Delta 5$-TAP. Therefore, we argue that the $\operatorname{Mrd} 1 \Delta 5$ mutation does not have a significant impact on the folding kinetics of the $27 \mathrm{~S}$ region of $35 \mathrm{~S}$ pre-rRNA.

We also attempted in vivo structure probing experiments with the SHAPE chemical 2-methylnicotinic acid imidazolide (NAI) ${ }^{46}$. Although this worked well for the stable cytoplasmic pre-40S 
complexes ${ }^{15}$, much to our surprise, incubating the cells with NAI significantly reduced the recovery of the $35 \mathrm{~S}$ and $27 \mathrm{~S}$ pre-rRNA species with TAP-tagged baits. As a result, we were unable to generate sufficient

NAI-probed pre-rRNA for high-throughput structure probing analyses. One potential reason is that these early assembly intermediates become unstable during the relatively long incubation times (5-10 min) required for optimal NAI modification.

One could argue that ex vivo analyses of the pre-ribosomes do not fully capture the state of the particle under physiological

a

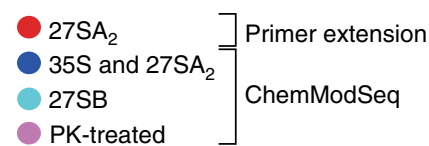
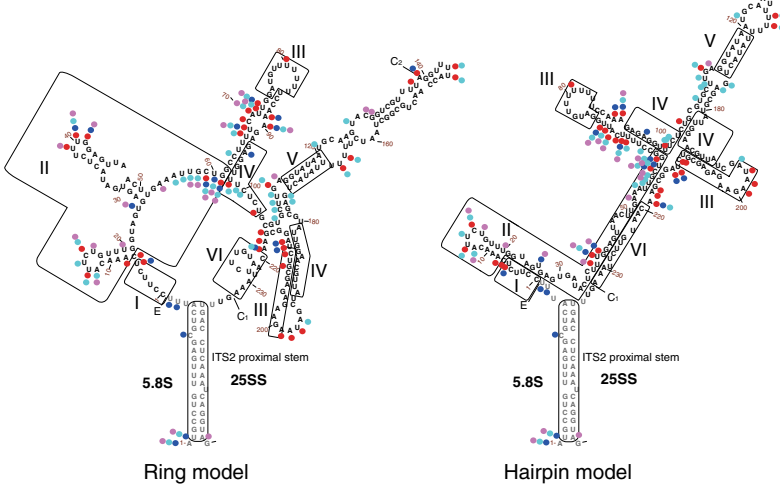

b

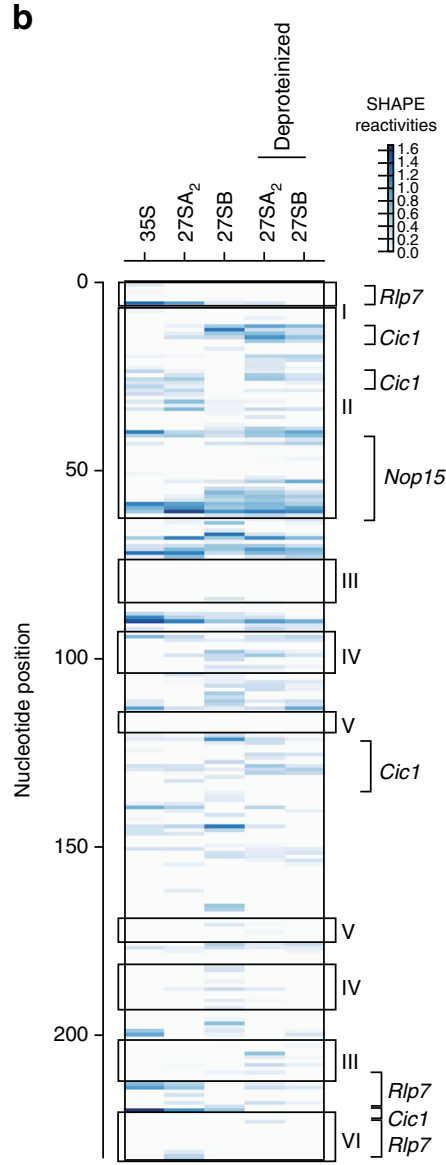

C $\frac{\text { DMSO }}{\text { Particle Deprotelinized }}$ A T C $\frac{1 \mathrm{M} 7}{\text { Particle Deprotenizized }}$

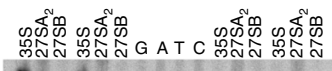
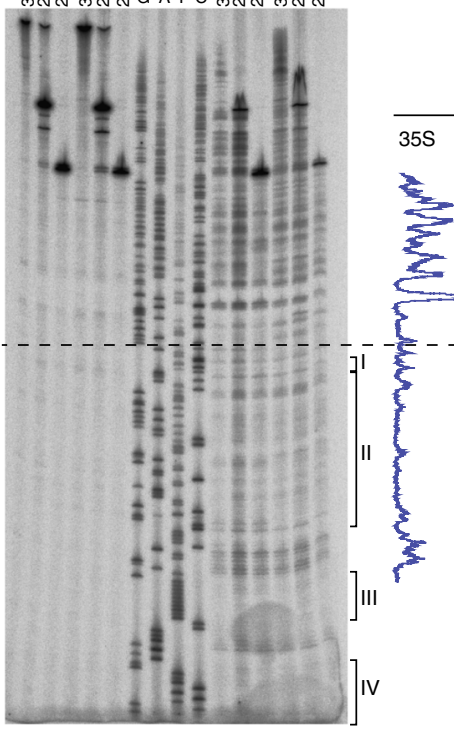

Particle
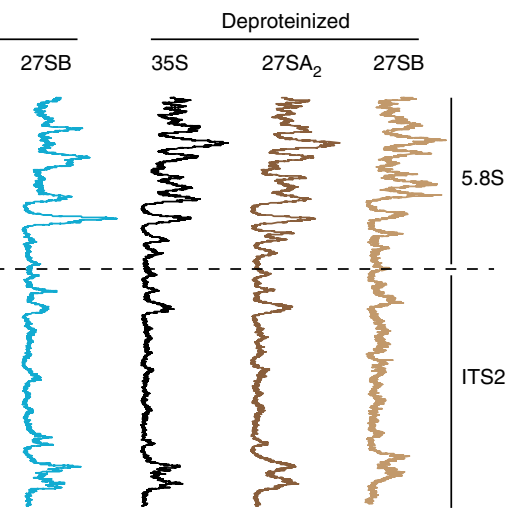

d

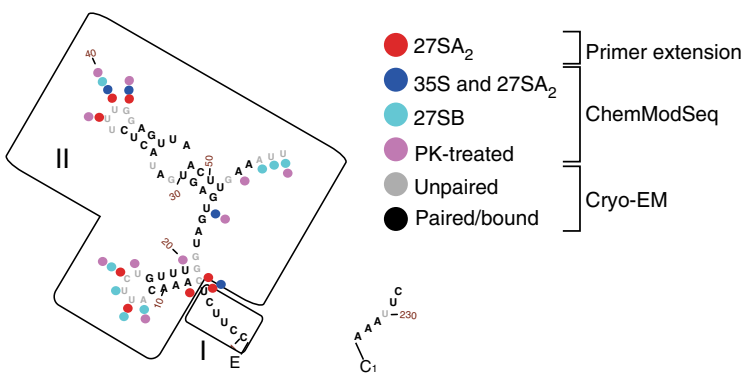


conditions. However, it is important to note that we purified the particles under conditions that are frequently used for tandem-affinity purification (TAP) of ribosome assembly intermediates as well as for cryo-EM studies. Therefore, the advantage of the ex vivo analyses is that we can directly compare our data with previously published structural and biochemical studies.

We report a detailed secondary structure analysis of the ITS2 region using the SHAPE reagent $1 \mathrm{M} 7$. In contrast to DMS used in previous studies ${ }^{44,45,47,48}$ that probes $\mathrm{A}^{\prime}$ 's and $\mathrm{C}^{\prime} \mathrm{s}, 1 \mathrm{M} 7$ allows to assay the flexibility of all four nucleotides. Our data indicate that ITS2 forms a very compact and helical structure, since 1M7 reactivity was generally much lower in ITS2 than in other regions, for example, 5.8S (Fig. 6c). Our data provides experimental support for a recently proposed secondary structure that we refer to as the ring-pin model, that is largely based on phylogenetic analyses ${ }^{17}$. Furthermore, our results are in good agreement with the recent cryo-EM structure of a late pre-60S intermediate in which two fragments of ITS2 (Fig. 6d) were resolved ${ }^{12}$.

On the basis of the combined results of rRNA structure predictions, enzymatic and chemical probing experiments, and genetic analyses ${ }^{37-40}$, it has been proposed that ITS2 may undergo a conformational switch that would allow 27SB pre-rRNA to be cleaved at site $\mathrm{C}_{2}$. Our data provides no support for such a dramatic conformational change during nucleolar ribosome assembly, as ITS2 as well as its flanking regions that base-pair with $25 \mathrm{~S}$ adopt a similar conformation in all particles analyzed (Fig. 6). However, we cannot exclude that such a switch occurs co-transcriptionally.

Chemical probing analyses have revealed that depletion of $\mathrm{A}_{3}$ factors influence the DMS modification pattern of nucleotides near the $5^{\prime}$ end of ITS $2^{44,47}$. These data suggest that $A_{3}$ factors are involved in maintaining an open structure of ITS2 and that their release could re-structure ITS2, such as into the proposed compact hairpin structure. Our chemical probing data did not reveal dramatic differences in nucleotide flexibility of ITS2 upon deproteinization of the native $27 \mathrm{SA}_{2}$ and $27 \mathrm{SB}$ particles (Fig. 6). We did observe a significant reduction in the flexibility of $U_{6}$ in the $5^{\prime}$ ITS2 in our deproteinized samples, which appears to be contacted by $\mathrm{Cic1}$ and Rlp7 in the cryo-EM structure. We therefore consider it more likely that the $\mathrm{A}_{3}$ factors influence the ITS2 structure locally, rather than being involved in drastic structural rearrangements such as the proposed open (ring) to closed (hairpin) conformational switch. In agreement with this, the nucleotides that showed differential reactivity to DMS upon $\mathrm{A}_{3}$ factor depletion ${ }^{44,47}$ overlap with or are in close proximity to Cic1- and Rlp7-binding sites ${ }^{12,44,47}$. These nucleotides did not strongly react with $1 \mathrm{M} 7$ in our particles or the PK-treated samples. A plausible explanation for these discrepancies is that $1 \mathrm{M} 7$ reacts with the $2^{\prime} \mathrm{OH}$ of the ribose, whereas DMS probes the bases of unpaired $\mathrm{C}^{\prime} \mathrm{s}$ and $\mathrm{A}^{\prime} \mathrm{s}$. It is possible that, due to riboses being constrained by proteins binding at or near these nucleotides, they may not react with $1 \mathrm{M} 7$, whereas corresponding bases still can be modified by DMS.
Our analyses enabled us to determine the exact timing of major restructuring events in the $5.8 \mathrm{~S}$ rRNA sequence. Formation of 5.8S-25S base-pairing interactions very likely takes place during transcription as those regions that form these base-pairing interactions generally reacted poorly to $1 \mathrm{M} 7$ and showed no significant changes in reactivity in the particles analyzed (Fig. 7). Moreover, while in the $35 \mathrm{~S}$ and $27 \mathrm{SA}_{2}$ particles the $5.8 \mathrm{~S}$ region appeared largely unfolded, it was highly structured in the 27SB-containing Nsa2 particles, suggesting that $5.8 \mathrm{~S}$ undergoes major structural rearrangements during the conversion of $27 \mathrm{SA}_{2}$ to $27 \mathrm{SB}$ (Fig. 7). One possibility is that these restructuring events coincide with the binding of r-proteins to 5.8S. Our ChemModSeq data show that flexibility changes in some nucleotides are indeed PK-sensitive, hinting that this could be the case for some regions. The data do not allow us to determine what comes first: RNA folding followed by r-protein binding, or whether the r-proteins actually induce the folding of the RNA. However, the data do suggest that r-proteins are at least partially required to stabilize the more constrained structure of some nucleotides shortly after it has been formed. Consistent with this idea, deletion of L26/uL24 also increases the flexibility around helices $\mathrm{H} 6$ and $\mathrm{H} 7$ in the $5.8 \mathrm{~S} \mathrm{rRNA}^{49}$. Stable incorporation of $\mathrm{r}$-proteins that interact with the $5.8 \mathrm{~S}$ region (L26/uL24, L35/uL29, and L37/eL37) requires the $\mathrm{A}_{3}$ factors, and it was proposed that rRNA remodeling by $\mathrm{A}_{3}$ factors might generate the binding sites for these r-proteins, allowing their stable incorporation into the pre- $60 S^{27} . A_{3}$ proteins were abundantly detected in our $27 \mathrm{SA}_{2}$ and $27 \mathrm{SB}$ particles (Fig. 1). Yet depletion of Cic1 and Nop15 does not noticeably affect the in vivo structure of $5.8 \mathrm{~S}^{44}$, suggesting that $\mathrm{A}_{3}$ factors do not directly influence $5.8 \mathrm{~S}$ folding.

L17/uL22, L35/uL29, and L37/eL37 were recently shown to be essential for the association of the late B-factors ${ }^{48}$. Thus, our current working hypothesis is that the stable incorporation of these $5.8 \mathrm{~S}$ r-proteins into pre-60S complexes signals that $5.8 \mathrm{~S}$ rRNA folding has been successfully completed, paving the way for the assembly of the late B-factors and cleavage at site $\mathrm{C}_{2}$ (Fig. 7).

Recent work has indicated that many r-proteins bind early pre-60S complexes with low affinity and that their association gradually becomes more stable during later assembly steps. Interestingly, we frequently observed a decrease in flexibility of nucleotides that overlapped with r-protein-binding sites identified in the $60 \mathrm{~S}$ crystal structure ${ }^{34}$. The change in flexibility of these nucleotides was (largely) restored when the particles were deproteinized (PK-sensitive sites; PKSRP), indicating that the change in nucleotide flexibility was the result of protein-RNA interactions. These data therefore allowed us to speculate about the formation of r-protein-rRNA interactions during pre-60S assembly. The chemical probing data do not provide direct evidence that the observed changes in rRNA flexibility were indeed mediated by r-protein binding to the nucleotide. Indeed, there are a few alternative scenarios that could explain the appearance of $\mathrm{PK}$-sensitive sites. It is possible that $\mathrm{r}$-proteins binding in the vicinity or transient binding of ribosome assembly factors could

\footnotetext{
Fig. 6 ITS2 forms a very compact structure. a Overview of the various proposed secondary structure models for ITS2 ${ }^{17,40,41}$. Roman numerals indicate the regions discussed in the main text. Dots of different colors represent nucleotides with SHAPE reactivity $\geq 0.4$ in ITS2 primer extension data (red) and $35 \mathrm{~S}$ and $27 \mathrm{SA}_{2}$ particles (dark blue), 27SB particles (light blue), and deproteinized samples (magenta) based on ChemModSeq data. Note that the primer extension results for the $3^{\prime}$ end are not shown as this region showed high variability in reactivity. The known binding sites for assembly factors that interact with ITS2 are highlighted in the ring-pin model. $\mathbf{b}$ Heat map representing the ChemModSeq $1 \mathrm{M} 7$ reactivities. Each block indicates a single nucleotide. The darker the block the higher the 1M7 reactivity. c Primer extension analysis of the $5^{\prime}$ end of ITS2 and the $3^{\prime}$ end of 5.8 S region and line scans of the signal intensities of each lane. $\mathbf{d}$ RNA structure probing data agrees well with the cryo-EM structure of ITS2 fragments. Shown is the secondary structure of parts of ITS2 that were resolved by cryo-EM ${ }^{12}$. The black nucleotides in the sequence are nucleotides that are either base-paired or bound by assembly factors in the structure. Gray nucleotides are predicted to be single-stranded as they are not involved in Watson-Crick or Hoogsteen base-pairing interactions and are not predicted to be bound by proteins
} 


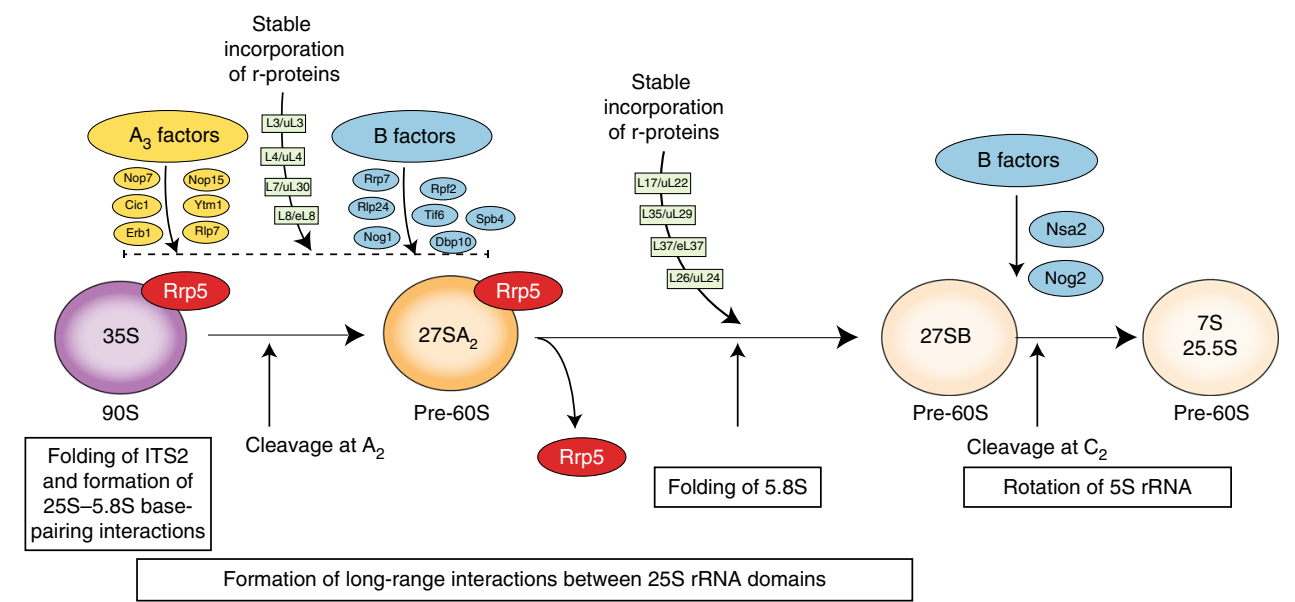

Fig. 7 Model of rRNA structural changes and pre-60S remodeling steps that take place during the nucleolar stages of 605 synthesis. ITS2 forms a highly compact structure in the nascent transcript. We predict that base-pairing interactions between 25S and 5.8S have already been formed cotranscriptionally. Major rRNA restructuring events occur in pre-ribosomal complexes during the conversion of 27SA 2 pre-rRNA to $27 \mathrm{SB}$ pre-rRNA. These include the compaction of the $5.8 \mathrm{~S}$ region and formation of long-range interactions in the $25 \mathrm{~S}$ sequence. All these changes occur after Rrp 5 dissociates and in the presence of $A_{3}$ and early $B$ factors. After $C_{2}$ cleavage, the $5 S$ rRNA rotates to adopt its final structure ${ }^{64}$

induce the observed change in flexibility. For example, we observed changes in the binding sites for L42/eL42, which we were unable to detect by MS and which were found to be mostly enriched in the late (Arx1) pre-60S complexes and the mature $80 \mathrm{~S}$ ribosomes ${ }^{36}$. Therefore, it is plausible that, in some cases, the observed changes in flexibility could be the result of another protein (such as an assembly factor) occupying these sites.

It has been suggested that assembly of pre-60S particles takes place in an hierarchical fashion and that the structural domains are formed in $5^{\prime}-3^{\prime}$ directionality (or at least some regions in the $5^{\prime}$ domain need to be assembled before the formation of $3^{\prime}$ domains can take place) ${ }^{50}$. This model proposed that the convex solvent side of the $60 \mathrm{~S}$ starts to form first followed by the polypeptide exit tunnel and finishing with the central protuberance. A large number of $\mathrm{r}$-proteins interact with multiple domains of the $25 \mathrm{~S} \mathrm{rRNA}^{34}$ and therefore, intuitively, it seems logical that binding of r-proteins must affect the folding of multiple domains. It is conceivable that long-range interactions could already be established at a very early stage of assembly and that several (parallel) assembly pathways might exist. Our analysis provided an opportunity to address this from the RNA perspective. By focussing on those changes in nucleotides that we predicted were the result of r-protein-rRNA interactions (PKSRP sites), we asked which domains of the 25S rRNA were most likely to undergo r-protein induced folding. Consistent with the proposed hierarchical assembly mode $l^{50}$, our results do indicate that most of the PKSRP sites in nucleolar pre-60S complexes concentrated on the solvent interface of the 60S subunit (domains I and II), whereas fewer predicted changes in protein-rRNA interactions were detected on the subunit interface side (domains III and IV) and the central protuberance, which were proposed to fold later (Fig. 3c). However, our results also support the idea that $r$-proteins forge contacts between many distant domains (such as interactions between domains I, II, III and V, VI) at a very early stage.

The next challenges will be to determine what role assembly factors play in directing these folding steps, and to generate detailed maps of the $60 \mathrm{~S}$ rRNA-folding pathways. We expect that (near) atomic resolution cryo-EM, combined with RNA structure probing and crystallographic data, will greatly accelerate our understanding of the dynamics of ribosome assembly.

\section{Methods}

Yeast strains. S. cerevisiae strain BY4741 (MATa; his $3 \Delta 1$; leu2 $\Delta 0$; met15 0 ; ura $3 \Delta 0$ ) was used as the parental strain. The TAP-tagged Rrp5 and Nsa2 strains were taken from the yeast TAP-fusion open reading frame (ORF) collection (GE healthcare) and were grown in YPDA media ( $2 \%$ glucose, $1 \%$ yeast extract, and $2 \%$ peptone) (Formedium). The TAP-tagged Mrd1 mutant was previously described $^{51}$ as ASY055. ASY055 cells were grown overnight in YPGA media ( $1 \%$ yeast extract, $2 \%$ peptone, $2 \%$ galactose, and $2 \%$ raffinose), shifted to YPDA media, and grown for $8 \mathrm{~h}$ at $30^{\circ} \mathrm{C}$ to an optical density $\left(\mathrm{OD}_{600}\right)$ of 1 .

Synthesis of 1M7. Synthesis of $1 \mathrm{M} 7$ was performed as previously described ${ }^{32,52}$ Briefly, sodium hydride ( $60 \%$ dispersion in mineral oil, 1.4 equiv, $5.72 \mathrm{mmol}, 229$ $\mathrm{mg}$ ) was suspended in $13 \mathrm{ml}$ of dimethylformamide (DMF anhydrous grade from Sigma-Aldrich) under a nitrogen atmosphere and stirred for $5 \mathrm{~min}$. 4-Nitroisatoic anhydride (1 equiv, $4.08 \mathrm{mmol}, 850 \mathrm{mg})$ in DMF $(9 \mathrm{ml})$ and then methyl iodide (1.05 equiv, $4.29 \mathrm{mmol}, 609 \mathrm{mg}, 270 \mu \mathrm{l}$ ) were added dropwise, and the reaction stirred for $24 \mathrm{~h}$. The reaction mixture was added to partially frozen $1 \mathrm{M} \mathrm{HCl}(150 \mathrm{ml})$ forming a yellow precipitate. This was recovered by filtration, washed with water, then ether and dried under vacuum for $14 \mathrm{~h}$ to yield crude product. This was then triturated from ether/pentane and dried under vacuum for a further $14 \mathrm{~h}$ to yield 1-methyl-7-nitroistoic anhydride $(585 \mathrm{mg}, 64 \%$; Supplementary Data 4).

Immunoprecipitation and chemical modification of rRNA. Cells were collected at $\mathrm{OD}_{600} \sim 1.0$ and lysed in $4 \mathrm{~V} \mathrm{~g}^{-1}$ ice cold TMN150 buffer $(50 \mathrm{mM}$ Tris $\mathrm{pH} 8$, $150 \mathrm{mM} \mathrm{NaCl}, 0.1 \% \mathrm{NP}-40,1.5 \mathrm{mM} \mathrm{MgCl}_{2}$, and Roche protease inhibitor cocktail) by vortexing for $5 \mathrm{~min}$ with $3 \mathrm{~V}$ per g of $0.5 \mathrm{~mm}$ Zirkonia beads (Thistle $)^{53}$. The lysate was incubated with $400 \mu \mathrm{l}$ IgG Sepharose beads (Amersham) for $1 \mathrm{~h}$ at $4^{\circ} \mathrm{C}$. The beads were then washed three times 5 min with $10 \mathrm{ml} \mathrm{TMN} 150$ buffer at $4{ }^{\circ} \mathrm{C}$ and finally resuspended in $200 \mu \mathrm{l}$ TMN150 buffer. Subsequently, the beads were incubated with 1M7 (dissolved in DMSO, $12.5 \mathrm{mM}$ final) or DMSO (negative control) for $3 \mathrm{~min}$ at room temperature. The final concentration of DMSO was 5\%. To deproteinize the particles, complexes bound to IgG beads were resuspended in $200 \mu \mathrm{l}$ TMN150 buffer containing 1\% SDS and incubated with $2 \mu \mathrm{g}$ per $\mu \mathrm{l}$ PK for $3 \mathrm{~h}$ at $14^{\circ} \mathrm{C}$ prior to $1 \mathrm{M} 7$ modification. The RNA was phenol-chloroformextracted, ethanol-precipitated, and resolved on a $1 \%$ low-melting agarose gel (Seaplaque GTG agarose, Lonza). The pre-rRNA were subsequently excised from the gel and extracted by melting the agarose melted at $65^{\circ} \mathrm{C}$ with five gel volumes of extraction buffer (10 mM Tris- $\mathrm{HCl}, 1 \mathrm{mM}$ EDTA, $\mathrm{pH}$ 7.5). RNA was subsequently purified by phenol-chloroform extraction and ethanol-precipitated. Purified $27 \mathrm{~S}$ pre-rRNAs were quantified on a 2100 bioanalyzer (Agilent) using an RNA Nano 6000 assay. All experiments were performed at least twice, and for each particle, we included separate control experiments. This was performed to minimize variability in coverage profiles between modified and control experiments.

Primer extension analyses. Reverse transcription was performed using Superscript III (Invitrogen) with $8 \mathrm{ng}$ of purified rRNA and ${ }^{32} \mathrm{P}$-radiolabeled oligonucleotides. Samples were incubated at $45^{\circ} \mathrm{C}$ for $45 \mathrm{~min}$, after which $1 \mu \mathrm{l}$ of Exonuclease I (NEB) was added, followed by a 30 -min incubation at $37^{\circ} \mathrm{C}$ 
Reactions were stopped by the addition of $5 \mu \mathrm{l}$ of RNA loading dye (NEB). Sequencing ladders were prepared using a plasmid containing an rDNA repeat (pBS-rDNA) and Sequenase v2.0 (USB/Affymetrix). Samples were resolved on a $6 \%$ polyacrylamide/ $8 \mathrm{M}$ urea gels. Band intensities were quantified using the FLA5100 phosphoimager system (Fuji). Signal decay correction was done using exponential function ( $b=0.999$ for DMSO and $b=0.995$ for 1 M7 treated samples). Structural reactivities were calculated by subtracting band intensity in the control lane from that of corresponding nucleotide in 1M7 lane. All negative values were set to zero. All values were $2-8 \%$ normalized. 1 M7/DMSO ratios were calculated and reactivities of nucleotides with $1 \mathrm{M} 7 / \mathrm{DMSO}<1.5$ were also set to zero to avoid false-positives.

Oligonucleotides used for primer extensions were:

ITS2_143-162 (5'-GATTAGCCGCAGTTGGTAAA-3'), ITS2_58_77 (5'-CAT CCAATGAAAAGGCCAGC-3'), ITS2nt162 (5'-GATTAGCCGCAGTTGGTAA A-3'), and 25Snt47 (5'-GATATGCTTAAGTTCAGC-3').

Northern blot analysis. Northern blot analysis was performed essentially as described ${ }^{54}$, using the following oligonucleotides:

RDN1c (5'-ATGAAAACTCCACAGTG-3')

RDN1e (5'-GGCCAGCAATTTCAAGT-3')

\section{ChemModSeq library preparation and sequence data analysis. ChemModSeq} libraries were prepared as described by Hector et al. ${ }^{15}$ A detailed overview of all the adapter sequences and primers used is provided in Supplementary Table 1. Individual libraries were pooled based on concentration and barcoding, and paired-end sequencing was performed on HiSeq systems by Edinburgh Genomics (Edinburgh). Raw data processing was carried out using the pyCRAC software package ${ }^{55}$ and our ChemModSeq pipeline (see Data and code availability section below). Data from replicate experiments were summed to increase the total coverage. The ChemModSeqPipeline python script runs the complete data processing pipeline. We initially used the TCP_EM algorithm ${ }^{15}$ for identifying 1M7-modified nucleotides. However, our recent comparative analyses showed that, although this algorithm has a high specificity, it generally calls a much lower number of nucleotides modified compared to our SHAPE reactivity script ${ }^{56}$, which is why we decided not to use it for these analyses. In this manuscript, we calculated SHAPE reactivities according to the StructureFold method ${ }^{33}$ using the CalculateSHAPE_reactivities.py script.

The ChemModSeq pipeline first trims the adapter sequences from the reads using Flexbar ${ }^{57}$ followed by demultiplexing of the samples using pyBarcodeFilter from the pyCRAC package. This removed the barcode information in the forward reads except for the last random nucleotide, which was removed using the TrimNucs.py script. The random barcode information (seven random nucleotides) was subsequently used to collapse the data (pyFastqDuplicateRemover.py). The resulting fasta output files were mapped to the yeast rDNA sequence using novoalign 2.04 . The pyReadCounter.py script was subsequently used to generate gene transfer format (GTF) interval files from the novo files that contained merged coordinates of read pairs that mapped to the $35 \mathrm{~S}$ within 1000 nucleotides from each other. Only properly paired reads were considered for the analyses. The resulting GTF files were then used to generate pileups containing drop-off counts and coverage information for the $35 \mathrm{~S}$ and $27 \mathrm{~S}$ sequences (using pyGTF2sgr.py). These count data were subsequently used to calculate SHAPE reactivities using the CalculateSHAPE_reactivities.py script, which also applies $2-8 \%$ normalization to scale the values between 0 and $\sim 2$. The $\triangle$ SHAPE reactivities were calculated using python code from the Weeks lab ${ }^{35}$. The $\triangle$ SHAPE values for the 27SB region in each sample analyzed is provided in Supplementary Data 2.

Mass spectrometry. Rrp5p-ProtA, Nsa2-ProtA, Fun12-ProtA, and controls (W303a, ProtA-tag expressed alone) were grown in YPD, collected in logarithmic phase $\left(\mathrm{OD}_{600} 0.6-0.85\right)$, and cryo-lysed as previously described ${ }^{58}$. The affinity purifications were performed in technical triplicates for samples and singulate for controls. The purification of Rrp5-ProtA, Nsa2-ProtA was performed in RNP150 (20 mM HEPES-KOH pH 7.4, $110 \mathrm{mM} \mathrm{KOAc,} \mathrm{0.5 \%} \mathrm{Triton} \mathrm{X-100,} \mathrm{0.1 \%} \mathrm{Tween-20,}$ $1: 100$ solution $\mathrm{P}, 1: 5000$ antifoam $\mathrm{A}$, and $150 \mathrm{mM} \mathrm{NaCl}$ ) and purification of Fun12-PrA was performed in TBT150 (same as RNP150 supplemented with $2 \mathrm{mM}$ $\mathrm{MgCl}_{2}, 1: 1000$ DTT) as described elsewhere ${ }^{58}$.

Briefly, $0.5 \mathrm{~g}$ of slightly thawed, cryo-lysed cell powder was vortex-resuspended in nine volumes of extraction buffer and further homogenized using a polytron $\left(30^{\prime \prime}\right.$ on ice). The centrifuged-cleared sample $\left(3200 \times \mathrm{g}, 10^{\prime}, 4^{\circ} \mathrm{C}\right)$ was incubated $\left(30^{\prime}\right.$, slow rotation, $4^{\circ} \mathrm{C}$ ) with $7.5 \mathrm{mg}$ of Dynabeads conjugated with rabbit IgG (160 $\mathrm{\mu g}$ IgG per mg bead) pre-equilibrated in extraction buffer. Prior to on-bead digestion, the beads were washed 10 times with extraction buffer; once with 100 $\mathrm{mM} \mathrm{NH} \mathrm{H}_{4} \mathrm{OAc} / 0.1 \mathrm{mM} \mathrm{MgCl} / 0.1 \%$ Tween-20 (room temperature, slow rotation, 5'); four times with $100 \mathrm{mM} \mathrm{NH}_{4} \mathrm{OAc} / 0.1 \mathrm{mM} \mathrm{MgCl}_{2}$ (three times fast and once at room temperature, slow rotation, $5 \mathrm{~min}$ ); and once with $20 \mathrm{mM}$ Tris- $\mathrm{HCl}(\mathrm{pH} \mathrm{8.0)}$.

Subsequent to single-step affinity purification (ssAP) purification, one-tenth of each affinity purification sample was eluted $\left(0.5 \mathrm{M} \mathrm{NH}_{4} \mathrm{OH}\right)$ and resolved on $4-12 \%$ gradient Bis-Tris NuPAGE gels for silver staining. The remaining samples were on-bead trypsin digested in a volume of $50 \mu \mathrm{l}(20 \mu \mathrm{g}$ per ml trypsin (Sigma, proteomics grade) in $20 \mathrm{mM}$ Tris- $\mathrm{HCl}(\mathrm{pH} 8.0), 37^{\circ} \mathrm{C}, 900$ r.p.m., $16-20 \mathrm{~h}$, and stopped with $2 \%$ formic acid ${ }^{59}$ ) and analyzed by tandem MS as described previously ${ }^{58}$.

Briefly, dried tryptic digests were zip tipped (Millipore, as per supplier recommendations) and loaded onto a C-18 reverse-phase capillary column $(15 \mathrm{~cm} \times 75 \mu \mathrm{m}$ (Length $(L) \times$ internal diameter (i.d.)); $5 \mu \mathrm{m}$ particles, $300 \AA$ ) for electrospray ionization time-of-flight MS. Ionization was performed by a Proxeon nanoelectrospray Flex ion source set to $1.3-1.7 \mathrm{kV}$. Chromatographic separation of peptides was performed at $250^{\circ} \mathrm{C}$ on an Easy-nLC II system (Proxeon Biosystems) at $300 \mathrm{nl}$ per $\mathrm{min}$, first over a gradient $(20 \mathrm{~min}$ ) from $95 \%$ solvent $\mathrm{A}$ (water-0.1\% formic acid) to $25 \%$ B ( $100 \%$ acetonitrile, $0.1 \%$ formic acid); then to $45 \%$ B (40 $\mathrm{min})$; and finally to $80 \% \mathrm{~B}(10 \mathrm{~min})$. Parent ion scans were performed in the HCD cell of the Q-Exactive (Thermo Fisher Scientific) over a mass range of 360-2000 m/ $\mathrm{z}$ at a resolving power of 70000 . The automatic gain control (AGC) target and maximum ion fill time (IT) were set to $1 \times 10^{6}$ and $100 \mathrm{~ms}$ respectively. Fragments were obtained with a normalized collision energy of 27 , an intensity threshold of $1.2 \times 10^{4}$, and underfill of $0.9 \%$. Data-dependent mode was used, in which 14 most intense precursor ions were isolated with a dynamic exclusion window of $15 \mathrm{~s}$. MS/ MS was acquired using a resolution of 17500 , an AGC target of $1 \times 10^{5}$, and a maximum IT of $50 \mathrm{~ms}$. Data were processed with Thermo Excalibur to generate a raw file. Mascot search server (version 2.3.02 ${ }^{60}$ ) was used with a parent tolerance of 10 p.p.m. for precursor ions, $0.52 \mathrm{Da}$ for fragments, and only considering one possible missed cleavage as well as a mass change of +16 for methionine oxidations in the mass calculation. Data were searched against $S$. cerevisiae database of NCBI (txid4932, 20140813 release) and analyzed in Scaffold (version 3.6.4). The threshold and false discovery rates were set to $80 \%$ and $0.37 \%$, respectively, for peptides, and to $95 \%$ (1 peptide minimum) and $1.9 \%$, respectively, for proteins.

Exclusive spectrum counts (ESCs) were used for analysis to discriminate against peptides shared between paralog proteins. For each prey, the highest values obtained in the controls were removed from those of the samples during analysis. Preys were defined as legitimate if they had at least two significantly enriched spectrum counts in at least two of the three replicates and selected for semi-quantitative analysis. The preys not selected for analysis are listed in Supplementary Data 1. For semi-quantitative analysis, exclusive spectral counts for L3 were used to normalize the data because it enters early during ribosome biogenesis and it was found in the three bait proteins in similar counts ${ }^{50,61,62}$ Semi-quantitative results were color-coded into four categories, high confidence and quantifiable (in red, $>10 \mathrm{ESC}, 3 / 3$ ); highly probable but not quantifiable (in orange, 7.5-9.9ESC, in 2/3); probable but not quantifiable (in yellow, 5-7.49ESC, in 2/3); and some evidence (in gray, 2-4.9ESC, 2/3). Standard deviations were calculated for the triplicates. Results are shown in alphabetical order and by categories (Supplementary Data 1).

Code availability. The scripts used for processing these data, a list of dependencies as well as a pdf file explaining our library design can be found on bitbucket.org/ sgrann/chemmodseqpipeline.

Data availability. The ChemModSeq sequencing data is available from the Gene Expression Omnibus under accession number GSE83821. The data that support the findings of this study are available from the corresponding author upon request.

Received: 29 June 2016 Accepted: 26 July 2017 Published online: 28 September 2017

\section{References}

1. Trapman, J., Retèl, J. \& Planta, R. J. Ribosomal precursor particles from yeast Exp. Cell Res. 90, 95-104 (1975).

2. Mougey, E. B. et al. The terminal balls characteristic of eukaryotic rRNA transcription units in chromatin spreads are rRNA processing complexes. Genes Dev. 7, 1609-1619 (1993).

3. Zhang, J. et al. Assembly factors Rpf2 and Rrs1 recruit 5S rRNA and ribosoma proteins rpL5 and rpL11 into nascent ribosomes. Genes Dev. 21, 2580-2592 (2007).

4. Geerlings, T. H., Vos, J. C. \& Raue, H. A. The final step in the formation of $25 \mathrm{~S}$ rRNA in Saccharomyces cerevisiae is performed by $5^{\prime}-->3^{\prime}$ exonucleases. RNA 6, 1698-1703 (2000).

5. Allmang, C. et al. Functions of the exosome in rRNA, snoRNA and snRNA synthesis. EMBO J. 18, 5399-5410 (1999).

6. Thomson, E. \& Tollervey, D. The final step in $5.8 \mathrm{~S}$ rRNA processing is cytoplasmic in Saccharomyces cerevisiae. Mol. Cell Biol. 30, 976-984 (2010).

7. la Cruz de, J., Karbstein, K. \& Woolford, J. L. Functions of ribosomal proteins in assembly of eukaryotic ribosomes in vivo. Annu. Rev. Biochem. 84, 93-129 (2015).

8. Shajani, Z., Sykes, M. T. \& Williamson, J. R. Assembly of bacterial ribosomes Annu. Rev. Biochem. 80, 501-526 (2011). 
9. Weis, F. et al. Mechanism of eIF6 release from the nascent $60 \mathrm{~S}$ ribosomal subunit. Nat. Struct. Mol. Biol. 22, 914-919 (2015).

10. Ulbrich, C. et al. Mechanochemical removal of ribosome biogenesis factors from nascent 60 S ribosomal subunits. Cell 138, 911-922 (2009).

11. Matsuo, Y. et al. Coupled GTPase and remodelling ATPase activities form a checkpoint for ribosome export. Nature 505, 112-116 (2013).

12. $\mathrm{Wu}, \mathrm{S}$. et al. Diverse roles of assembly factors revealed by structures of late nuclear pre-60S ribosomes. Nature 534, 133-137 (2016).

13. Greber, B. J. et al. Insertion of the biogenesis factor Reil probes the ribosomal tunnel during 60S maturation. Cell 164, 91-102 (2016).

14. Bassler, J. et al. A network of assembly factors is involved in remodeling rRNA elements during preribosome maturation. J. Cell Biol. 210, 169-170 (2015).

15. Hector, R. D. et al. Snapshots of pre-rRNA structural flexibility reveal eukaryotic $40 \mathrm{~S}$ assembly dynamics at nucleotide resolution. Nucleic Acids Res. 42, 12138-12154 (2014).

16. Lebaron, S. et al. Rrp5 Binding at multiple sites coordinates pre-rRNA processing and assembly. Mol. Cell 52, 707-719 (2013).

17. Coleman, A. W. Nuclear rRNA transcript processing versus internal transcribed spacer secondary structure. Trends. Genet. 31, 157-163 (2015)

18. Dragon, F. et al. A large nucleolar U3 ribonucleoprotein required for $18 \mathrm{~S}$ ribosomal RNA biogenesis. Nature 417, 967-970 (2002).

19. Lebreton, A., Saveanu, C., Decourty, L., Jacquier, A. \& Fromont-Racine, M. Nsa2 is an unstable, conserved factor required for the maturation of $27 \mathrm{SB}$ pre-rRNAs. J. Biol. Chem. 281, 27099-27108 (2006).

20. Segerstolpe, A. et al. Multiple RNA interactions position Mrd1 at the site of the small subunit pseudoknot within the $90 \mathrm{~S}$ pre-ribosome. Nucleic Acids Res. 41, 1178-1190 (2013).

21. Lundkvist, P. et al. Mrd1p is required for release of base-paired U3 snoRNA within the preribosomal complex. Mol. Cell. Biol. 29, 5763-5774 (2009).

22. Hierlmeier, T. et al. Rrp5p, Noclp and Noc2p form a protein module which is part of early large ribosomal subunit precursors in S. cerevisiae. Nucleic Acids Res. 41, 1191-1210 (2012)

23. Babiano, R. et al. Yeast ribosomal protein L7 and its homologue Rlp7 are simultaneously present at distinct sites on pre-60S ribosomal particles. Nucleic Acids Res. 41, 9461-9470 (2013).

24. Jakovljevic, J. et al. Ribosomal proteins L7 and L8 function in concert with six $\mathrm{A}_{3}$ assembly factors to propagate assembly of domains I and II of 25S rRNA in yeast 60 S ribosomal subunits. RNA 18, 1805-1822 (2012).

25. Venema, J. \& Tollervey, D. RRP5 is required for formation of both $18 \mathrm{~S}$ and $5.8 \mathrm{~S}$ rRNA in yeast. EMBO J. 15, 5701-5714 (1996).

26. Lebreton, A. et al. $60 \mathrm{~S}$ ribosomal subunit assembly dynamics defined by semi-quantitative mass spectrometry of purified complexes. Nucleic Acids Res. 36, 4988-4999 (2008).

27. Sahasranaman, A. et al. Assembly of Saccharomyces cerevisiae 60S ribosomal subunits: role of factors required for $27 \mathrm{~S}$ pre-rRNA processing. EMBO J. 30, 4020-4032 (2011)

28. Talkish, J., Zhang, J., Jakovljevic, J., Horsey, E. W. \& Woolford, J. L. Hierarchical recruitment into nascent ribosomes of assembly factors required for 27SB pre-rRNA processing in Saccharomyces cerevisiae. Nucleic Acids Res. 40, 8646-8661 (2012)

29. Mager, W. H. et al. A new nomenclature for the cytoplasmic ribosomal proteins of Saccharomyces cerevisiae. Nucleic Acids Res. 25, 4872-4875 (1997).

30. Ban, N. et al. A new system for naming ribosomal proteins. Curr. Opin. Struct. Biol. 24, 165-169 (2014)

31. Gamalinda, M. \& Woolford, J. L. Paradigms of ribosome synthesis: lessons learned from ribosomal proteins. Translation 3, e975018 (2015).

32. Mortimer, S. A. \& Weeks, K. M. A fast-acting reagent for accurate analysis of RNA secondary and tertiary structure by SHAPE chemistry. J. Am. Chem. Soc. 129, 4144-4145 (2007).

33. Tang, Y. et al. StructureFold: genome-wide RNA secondary structure mapping and reconstruction in vivo. Bioinformatics 31, 2668-2675 (2015).

34. Ben-Shem, A. et al. The structure of the eukaryotic ribosome at $3.0 \AA$ resolution. Science 334, 1524-1529 (2011).

35. Smola, M. J., Calabrese, J. M. \& Weeks, K. M. Detection of RNA-protein interactions in living cells with SHAPE. Biochemistry 54, 6867-6875 (2015).

36. Ohmayer, U. et al. Studies on the assembly characteristics of large subunit ribosomal proteins in S. cerevisae. PLoS ONE 8, e68412 (2013).

37. Babiano, R. \& la Cruz de, J. Ribosomal protein L35 is required for $27 \mathrm{SB}$ pre-rRNA processing in Saccharomyces cerevisiae. Nucleic Acids Res. 38, 5177-5192 (2010)

38. Talkish, J., Campbell, I. W., Sahasranaman, A., Jakovljevic, J. \& Woolford, J. L. Ribosome assembly factors Pwp1 and Nop12 are important for folding of 5.8S rRNA during ribosome biogenesis in Saccharomyces cerevisiae. Mol. Cell Biol. 34, 1863-1877 (2014)

39. Joseph, N., Krauskopf, E., Vera, M. I. \& Michot, B. Ribosomal internal transcribed spacer 2 (ITS2) exhibits a common core of secondary structure in vertebrates and yeast. Nucleic Acids Res. 27, 4533-4540 (1999).
40. Yeh, L. C. \& Lee, J. C. Structural analysis of the internal transcribed spacer 2 of the precursor ribosomal RNA from Saccharomyces cerevisiae. J. Mol. Biol. 211, 699-712 (1990).

41. Côté, C. A., Greer, C. L. \& Peculis, B. A. Dynamic conformational model for the role of ITS2 in pre-rRNA processing in yeast. RNA 8, 786-797 (2002).

42. van Nues, R. W. et al. Evolutionarily conserved structural elements are critical for processing of internal transcribed spacer 2 from Saccharomyces cerevisiae precursor ribosomal RNA. J. Mol. Biol. 250, 24-36 (1995).

43. van der Sande, C. A. et al. Functional analysis of internal transcribed spacer 2 of Saccharomyces cerevisiae ribosomal DNA. J. Mol. Biol. 223, 899-910 (1992).

44. Granneman, S., Petfalski, E. \& Tollervey, D. A cluster of ribosome synthesis factors regulate pre-rRNA folding and $5.8 \mathrm{~S}$ rRNA maturation by the Rat1 exonuclease. EMBO J. 30, 4006-4019 (2011).

45. Dembowski, J. A., Kuo, B. \& Woolford, J. L. Has1 regulates consecutive maturation and processing steps for assembly of $60 \mathrm{~S}$ ribosomal subunits. Nucleic Acids Res. 41, 7889-7904 (2013).

46. Spitale, R. C. et al. RNA SHAPE analysis in living cells. Nat. Chem. Biol. 9 18-20 (2013).

47. Dembowski, J. A., Ramesh, M., McManus, C. J. \& Woolford, J. L. Identification of the binding site of Rlp7 on assembling 60S ribosomal subunits in Saccharomyces cerevisiae. RNA 19, 1639-1647 (2013).

48. Gamalinda, M. et al. Yeast polypeptide exit tunnel ribosomal proteins L17, L35 and L37 are necessary to recruit late-assembling factors required for 27SB pre-rRNA processing. Nucleic Acids Res. 41, 1965-1983 (2012).

49. Babiano, R., Gamalinda, M., Woolford, J. L. \& la Cruz de, J. Saccharomyces cerevisiae ribosomal protein L26 is not essential for ribosome assembly and function. Mol. Cell Biol. 32, 3228-3241 (2012).

50. Gamalinda, M. et al. A hierarchical model for assembly of eukaryotic $60 \mathrm{~S}$ ribosomal subunit domains. Genes Dev. 28, 198-210 (2014).

51. Segerstolpe, A. et al. Multiple RNA interactions position Mrd1 at the site of the small subunit pseudoknot within the $90 \mathrm{~S}$ pre-ribosome. Nucleic Acids Res. 41, 1178-1190 (2012).

52. Turner, R., Shefer, K. \& Ares, M. Safer one-pot synthesis of the 'SHAPE' reagent 1-methyl-7-nitroisatoic anhydride (1m7). RNA 19, 1857-1863 (2013).

53. Granneman, S., Petfalski, E., Swiatkowska, A. \& Tollervey, D. Cracking pre-40S ribosomal subunit structure by systematic analyses of RNA-protein cross-linking. EMBO J. 29, 2026-2036 (2010).

54. Beyer, A. L., Segerstolpe, A., Lundkvist, P., Osheim, Y. N. \& Wieslander, L. Mrdlp binds to pre-rRNA early during transcription independent of U3 snoRNA and is required for compaction of the pre-rRNA into small subunit processomes. Nucleic Acids Res. 36, 4364-4380 (2008).

55. Webb, S., Hector, R. D., Kudla, G. \& Granneman, S. PAR-CLIP data indicate that Nrd1-Nab3-dependent transcription termination regulates expression of hundreds of protein coding genes in yeast. Genome Biol. 15, R8 (2014).

56. Selega, A., Sirocchi, C., Iosub, I., Granneman, S. \& Sanguinetti, G. Robust statistical modeling improves sensitivity of high-throughput RNA structure probing experiments. Nat. Methods 14, 83-89 (2016).

57. Dodt, M., Roehr, J. T., Ahmed, R. \& Dieterich, C. FLEXBAR-flexible barcode and adapter processing for next-generation sequencing platforms. Biology $\mathbf{1}$, 895-905 (2012)

58. Oeffinger, M. et al. Comprehensive analysis of diverse ribonucleoprotein complexes. Nat. Methods 4, 951-956 (2007).

59. Gingras, A.-C., Gstaiger, M., Raught, B. \& Aebersold, R. Analysis of protein complexes using mass spectrometry. Nat. Rev. Mol. Cell Biol. 8, 645-654 (2007)

60. Rosado, I. V., Kressler, D. \& la Cruz de, J. Functional analysis of Saccharomyces cerevisiae ribosomal protein Rpl3p in ribosome synthesis. Nucleic Acids Res. 35, 4203-4213 (2007).

62. Piir, K., Tamm, T., Kisly, I., Tammsalu, T. \& Remme, J. Stepwise splitting of ribosomal proteins from yeast ribosomes by LiCl. PLoS ONE 9, e101561 (2014).

63. Bernier, C. R. et al. RiboVision suite for visualization and analysis of ribosomes Faraday Discuss. 169, 195-207 (2014).

64. Leidig, C. et al. $60 \mathrm{~S}$ ribosome biogenesis requires rotation of the $5 \mathrm{~S}$ ribonucleoprotein particle. Nat. Commun. 5, 3491 (2014)

\section{Acknowledgements}

This work was supported by grants from the Wellcome Trust to S.G. (091549) to E.B. (096997) and the Wellcome Trust Centre for Cell Biology core grant (092076). F.L. was supported by a short-term EMBO fellowship (ASTF 568-2012). L.W., S.B., and F.L. are supported by the Swedish Research Council. M.O. is supported by a Discovery Grant from the Natural Sciences and Engineering Research Council of Canada (RGPIN 386315-MO) and a Project Grant from Canadian Institutes of Health Research (PTJ-153313). N.D.-W. is supported by the Engineering and Physical Sciences Research Council. S.L.C. acknowledges support from a European Research Council Starting Grant (336935). Next-generation sequencing was carried out by Edinburgh Genomics, The University of Edinburgh. Edinburgh Genomics is partly 
supported through core grants from NERC (R8/H10/56), MRC (MR/K001744/1), and BBSRC (BB/J004243/1).

\section{Author contributions}

E.B., L.W., M.O., and S.G. designed the research. E.B., S.G., F.L., L.-C.A., S.B., R.v.N., and R.D.H. performed the experiments and analyzed the data. S.B. quantified the primer extension data and C.T. compared the probing data to cryo-EM and crystal structures. N.D.-W. and S.L.C. synthesized the 1M7. L.-C.A. and M.O. performed the mass spectrometry experiments and data analyses. All authors contributed to the writing of the manuscript.

\section{Additional information}

Supplementary Information accompanies this paper at doi:10.1038/s41467-017-00761-8.

Competing interests: The authors declare no competing financial interests.

Reprints and permission information is available online at http://npg.nature.com/ reprintsandpermissions/
Publisher's note: Springer Nature remains neutral with regard to jurisdictional claims in published maps and institutional affiliations.

(c) (i) Open Access This article is licensed under a Creative Commons Attribution 4.0 International License, which permits use, sharing, adaptation, distribution and reproduction in any medium or format, as long as you give appropriate credit to the original author(s) and the source, provide a link to the Creative Commons license, and indicate if changes were made. The images or other third party material in this article are included in the article's Creative Commons license, unless indicated otherwise in a credit line to the material. If material is not included in the article's Creative Commons license and your intended use is not permitted by statutory regulation or exceeds the permitted use, you will need to obtain permission directly from the copyright holder. To view a copy of this license, visit http://creativecommons.org/ licenses/by/4.0/.

(C) The Author(s) 2017 